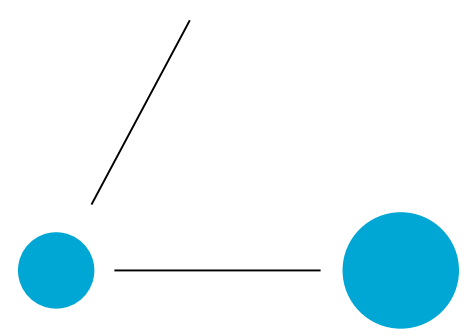

A N N A L E S HENRI LEBESGUE

\title{
VAHAGN NERSESYAN
}

\section{LARGE DEVIATIONS FOR THE NAVIER-STOKES EQUATIONS DRIVEN BY A WHITE-IN-TIME NOISE}

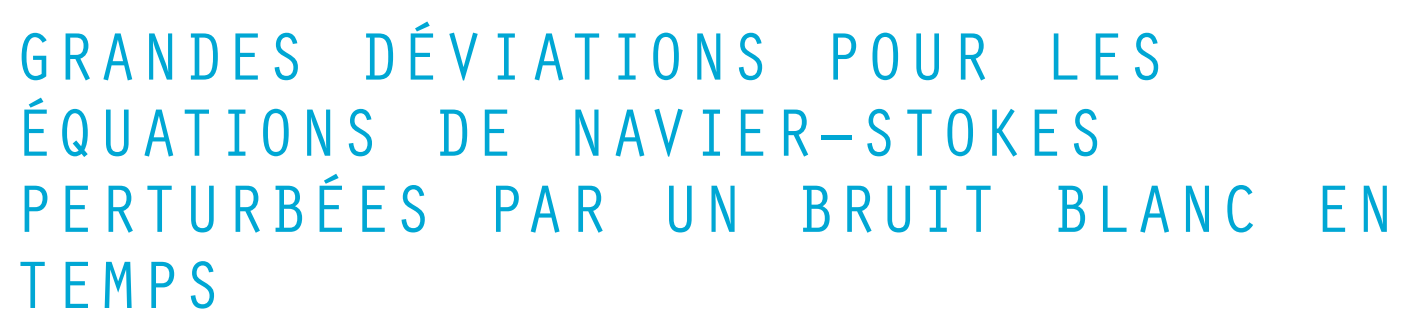

Abstract. - In this paper, we consider the 2D Navier-Stokes system driven by a white-in-time noise. We show that the occupation measures of the trajectories satisfy a large deviations principle, provided that the noise acts directly on all Fourier modes. The proofs are obtained by developing an approach introduced previously for discrete-time random dynamical systems, based on a Kifer-type criterion and a multiplicative ergodic theorem.

RÉSumÉ. - Dans cet article, nous étudions le système de Navier-Stokes en dimension deux perturbé par un bruit blanc en temps. Nous montrons un principe de grandes déviations pour les mesures empiriques des trajectoires sous l'hypothèse que tous les modes de Fourier sont excités par le bruit. La preuve utilise une approche introduite précédemment pour des

Keywords: Stochastic Navier-Stokes system, large deviations principle, occupation measures, multiplicative ergodicity.

2010 Mathematics Subject Classification: 35Q30, 60B12, 60F10, 37H15.

DOI: https://doi.org/10.5802/ahl.23

(*) The author thanks Davit Martirosyan and Armen Shirikyan for many discussions. This research was supported by the ANR grant NONSTOPS ANR-17-CE40-0006-02 and CNRS PICS grant Fluctuation theorems in stochastic systems. 
systèmes dynamiques aléatoires à temps discret, basée sur un critère de type Kifer et un théorème ergodique multiplicatif.

\section{Introduction}

We study the large deviations principle (LDP) for the 2D Navier-Stokes system for incompressible fluids:

$$
\partial_{t} u+\langle u, \nabla\rangle u-\nu \Delta u+\nabla p=f(t, x), \quad \operatorname{div} u=0,
$$

where $\nu>0$ is the viscosity of the fluid, $u=\left(u_{1}(t, x), u_{2}(t, x)\right)$ and $p=p(t, x)$ are unknown velocity field and pressure, $f$ is an external (random) force, and $\langle u, \nabla\rangle=$ $u_{1} \partial_{1}+u_{2} \partial_{2}$. Throughout this paper, we assume that the space variable $x=\left(x_{1}, x_{2}\right)$ belongs ${ }^{(1)}$ to the standard torus $\mathbb{T}^{2}=\mathbb{R}^{2} / 2 \pi \mathbb{Z}^{2}$. The problem is considered in the space of divergence-free vector fields with zero mean value

$$
H=\left\{u \in L^{2}\left(\mathbb{T}^{2}, \mathbb{R}^{2}\right): \operatorname{div} u=0 \text { in } \mathbb{T}^{2}, \int_{\mathbb{T}^{2}} u(x) \mathrm{d} x=0\right\}
$$

endowed with the $L^{2}$-norm $\|\cdot\|$. We assume that the force is of the form

$$
f(t, x)=h(x)+\eta(t, x),
$$

where $h \in H^{1}:=H^{1}\left(\mathbb{T}^{2}, \mathbb{R}^{2}\right) \cap H$ is a given function and $\eta$ is a white-in-time noise

$$
\eta(t, x)=\partial_{t} W(t, x), \quad W(t, x)=\sum_{j=1}^{\infty} b_{j} \beta_{j}(t) e_{j}(x) .
$$

Here $\left\{b_{j}\right\}$ is sequence of real numbers such that

$$
\mathfrak{B}_{1}=\sum_{j=1}^{\infty} \alpha_{j} b_{j}^{2}<\infty
$$

$\left\{\beta_{j}\right\}$ is a sequence of independent standard Brownian motions defined on a filtered probability space ${ }^{(2)}\left(\Omega, \mathcal{F},\left\{\mathcal{F}_{t}\right\}, \mathbb{P}\right)$, and $\left\{e_{j}\right\}$ is an orthonormal basis in $H$ consisting of the eigenfunctions of the Stokes operator $L=-\Delta$ with eigenvalues $\left\{\alpha_{j}\right\}$. As usual, projecting (1.1) to $H$, we eliminate the pressure and obtain an evolution equation for the velocity field ${ }^{(3)}$ (e.g., see [Lio69, Chapter 1, Section 6]):

$$
\dot{u}+B(u)+L u=h(x)+\eta(t, x),
$$

where $B(u)=\Pi(\langle u, \nabla\rangle u)$ and $\Pi$ is the orthogonal projection onto $H$ in $L^{2}$. This system is supplemented with the initial condition

$$
u(0)=u_{0} .
$$

Under these assumptions, problem (1.5), (1.6) admits a unique solution and defines a Markov family $\left(u_{t}, \mathbb{P}_{u}\right)$ parametrised by the initial condition $u=u_{0} \in H$. The

(1) The periodic boundary conditions are chosen to simplify the presentation. Similar results can be established in the case of a bounded domain with smooth boundary and Dirichlet boundary conditions.

${ }^{(2)}$ We assume that this space satisfies the usual conditions (see [KS91, Definition 2.25]).

${ }^{(3)}$ To simplify the notation, we shall assume that $\nu=1$. 
ergodic properties of this family are now well understood. In particular, it is known that $\left(u_{t}, \mathbb{P}_{u}\right)$ admits a unique stationary measure, which is exponentially mixing, provided that sufficiently many coefficients $b_{j}$ are non-zero (see the papers [FM95, KS00, EMS01, KS02, BKL02, HM06, Oda08] and the book [KS12]). A central limit theorem (CLT) for problem (1.5), (1.6) is established in [Kuk02, Shi06]. The LDP proved in the present paper is a natural extension of the CLT. Indeed, while the CLT describes the probability of small deviations of a time average of a functional from its mean value, the LDP quantifies the probability of large deviations.

Before formulating the main result of this paper, let us introduce some notation and definitions. We denote by $\mathcal{P}(H)$ the space of Borel probability measures on $H$ endowed with the topology of weak convergence. Given a measure $\nu \in \mathcal{P}(H)$, we set $\mathbb{P}_{\nu}(\Gamma)=\int_{H} \mathbb{P}_{u}(\Gamma) \nu(\mathrm{d} u)$ for any $\Gamma \in \mathcal{F}$ and consider the following family of occupation measures

$$
\zeta_{t}=\frac{1}{t} \int_{0}^{t} \delta_{u_{s}} \mathrm{~d} s, \quad t>0
$$

defined on the probability space $\left(\Omega, \mathcal{F}, \mathbb{P}_{\nu}\right)$. Here $\delta_{u}$ is the Dirac measure concentrated at $u \in H$. We shall say that a mapping $I: \mathcal{P}(H) \rightarrow[0,+\infty]$ is a good rate function if the level set $\{\sigma \in \mathcal{P}(H): I(\sigma) \leqslant \alpha\}$ is compact for any $\alpha \geqslant 0$. A good rate function $I$ is nontrivial if its effective domain $D_{I}=\{\sigma \in \mathcal{P}(H): I(\sigma)<\infty\}$ is not a singleton. For any numbers $\varkappa>0$ and $M>0$, we denote

$$
\Lambda(\varkappa, M)=\left\{\nu \in \mathcal{P}(H): \int_{H} e^{\varkappa\|v\|^{2}} \nu(\mathrm{d} v) \leqslant M\right\} .
$$

Main Theorem. - Assume that (1.4) holds and $b_{j}>0$ for all $j \geqslant 1$. Then for any numbers $\varkappa>0$ and $M>0$, the family $\left\{\zeta_{t}, t>0\right\}$ satisfies an LDP, uniformly with respect to $\nu \in \Lambda(\varkappa, M)$, with a non-trivial good rate function $I: \mathcal{P}(H) \rightarrow[0,+\infty]$ not depending on $\varkappa$ and $M$. More precisely, the following two bounds hold.

Upper bound. - For any closed subset $F \subset \mathcal{P}(H)$, we have

$$
\limsup _{t \rightarrow \infty} \frac{1}{t} \log \sup _{\nu \in \Lambda} \mathbb{P}_{\nu}\left\{\zeta_{t} \in F\right\} \leqslant-\inf _{\sigma \in F} I(\sigma) \text {. }
$$

Lower bound. - For any open subset $G \subset \mathcal{P}(H)$, we have

$$
\liminf _{t \rightarrow \infty} \frac{1}{t} \log \inf _{\nu \in \Lambda} \mathbb{P}_{\nu}\left\{\zeta_{t} \in G\right\} \geqslant-\inf _{\sigma \in G} I(\sigma) .
$$

Furthermore, $I$ is given by

$$
I(\sigma)=\sup _{V \in C_{b}(H)}\left(\int_{H} V(u) \sigma(\mathrm{d} u)-Q(V)\right), \quad \sigma \in \mathcal{P}(H),
$$

where $Q: C_{b}(H) \rightarrow \mathbb{R}$ is a 1-Lipschitz convex function such that $Q(C)=C$ for any $C \in \mathbb{R}$.

This type of large deviations results have been first established by Donsker and Varadhan [DV75] and later generalised by many others (see the books [FW84, DS89, DZ00] and the references therein). There are only a few works studying the large deviations behaviour of solutions of randomly forced PDEs as time goes to infinity. 
The case of the stochastic Burgers and Navier-Stokes equations is first studied in [Gou07a, Gou07b]. In these papers, the random perturbation is of the form (1.3) with the following restriction on the coefficients

$$
c j^{-\alpha} \leqslant b_{j} \leqslant C j^{-\frac{1}{2}-\varepsilon}, \quad \frac{1}{2}<\alpha<1, \varepsilon \in\left(0, \alpha-\frac{1}{2}\right] .
$$

Notice that the lower bound does not allow the sequence $\left\{b_{j}\right\}$ to converge to zero sufficiently fast, so the external force $f$ is irregular with respect to the space variable. This is not very natural from the physical point of view. The proof is based on a general sufficient condition established in [Wu01], and essentially uses the strong Feller property. The main novelty of our Main Theorem is that it proves an LDP without any lower bound on $\left\{b_{j}\right\}$ (so, in particular, we do not have a strong Feller property).

We use an approach introduced in the papers [JNPS15, JNPS18], where an LDP is established for a family of dissipative PDEs perturbed by a random kick force. The proofs of these papers are based on a Kifer type criterion for LDP and a study of the large-time behaviour of generalised Markov semigroups. These results have been later extended in [MN18a] to the case of the stochastic damped nonlinear wave equation driven by a spatially regular white noise. The main result of that paper is an LDP of local type. In the case of the Navier-Stokes system (1.5), although we follow a similar scheme, there are important differences in all the steps of the argument, coming from both the continuous-time nature of the system and the globalness of the LDP. Here we study the large-time asymptotics of the Feynman-Kac semigroup without any restriction on the smallness of the potential. One of the most important difficulties arises in the proof of the uniform Feller property. To establish this, we construct coupling processes using a new two parameter auxiliary equation (see (4.1)) which allows to have an appropriate Foiaş-Prodi estimate for the trajectories and a rapid exponential stabilisation for finite-dimensional projections.

Let us also mention that the multiplicative ergodic theorem we obtain for system (1.5) is of more general form and works for a larger class of functionals and initial measures (see Theorem 2.1).

It is a challenging open problem whether an LDP still holds for (1.5), (1.6) when the driving noise is highly degenerate (i.e., only a finite number of $b_{j}$ are non-zero in (1.3)). For the Navier-Stokes system in this degenerate situation, exponential mixing is established in [HM06] for white-in-time noise and in [KNS18] for a bounded noise satisfying some decomposability and observability hypotheses. Using these results and literally repeating the arguments of the proof of Theorem 5.4 in [MN18b], one can prove a level-1 LDP of local type.

The paper is organised as follows. In Section 2, we state a multiplicative ergodic theorem for the Navier-Stokes system and combine it with Kifer's criterion for non-compact spaces to prove the Main Theorem. In Sections 3 and 4, we check the conditions of an abstract result on large-time behaviour of generalised Markov semigroups. Section 5 is devoted to the proof of the multiplicative ergodicity. In the Appendix, we prove various a priori estimates for the solutions and recall the statement of the above-mentioned result for generalised Markov semigroups. 


\section{Notation}

We shall use the following standard notation.

- $H$ is the space defined by $(1.2), B_{H}(a, R)$ is the closed ball in $H$ of radius $R$ centred at $a$. When $a=0$, we write $B_{H}(R)$.

- $H^{1}=H^{1}\left(\mathbb{T}^{2}, \mathbb{R}^{2}\right) \cap H$, where $H^{1}\left(\mathbb{T}^{2}, \mathbb{R}^{2}\right)$ is the space of vector functions $u=\left(u_{1}, u_{2}\right)$ with components in the usual Sobolev space of order 1 on $\mathbb{T}^{2}$.

- $L^{\infty}(H)$ is the space of bounded Borel-measurable functions $f: H \rightarrow \mathbb{R}$ endowed with the norm $\|f\|_{\infty}=\sup _{u \in H}|f(u)|$.

- $C_{b}(H)$ is the space of continuous functions $f \in L^{\infty}(H)$.

- $L_{b}(H)$ is the space of functions $f \in C_{b}(H)$ for which the following norm is finite

$$
\|\psi\|_{L}=\|\psi\|_{\infty}+\sup _{u \neq v} \frac{|\psi(u)-\psi(v)|}{\|u-v\|} .
$$

- $\mathcal{V}$ is the space of functions $V: H \rightarrow \mathbb{R}$ for which there is an integer $N \geqslant 1$ and a function $F \in L_{b}\left(H_{N}\right)$ such that

$$
V(u)=F\left(\mathrm{P}_{N} u\right), \quad u \in H .
$$

Here $\mathrm{P}_{N}$ is the orthogonal projection in $H$ onto the space

$$
H_{N}=\operatorname{span}\left\{e_{1}, \ldots, e_{N}\right\}
$$

and $\left\{e_{j}\right\}$ is the orthonormal basis entering (1.3).

- For a given Borel-measurable function $\mathfrak{w}: H \rightarrow[1,+\infty]$, we denote by $C_{\mathfrak{w}}(H)$ (respectively, $\left.L_{\mathfrak{w}}^{\infty}(H)\right)$ the space of continuous (Borel-measurable) functions $f: H \rightarrow \mathbb{R}$ such that

$$
\|f\|_{L_{\mathfrak{w}}^{\infty}}=\sup _{u \in H} \frac{|f(u)|}{\mathfrak{w}(u)}<\infty .
$$

- $\mathcal{M}_{+}(H)$ is the set of non-negative finite Borel measures on $H$ endowed with the topology of the weak convergence. For $\mu \in \mathcal{M}_{+}(H)$ and $f \in C_{b}(H)$, we denote $\langle f, \mu\rangle=\int_{H} f(u) \mu(\mathrm{d} u)$.

- $\mathcal{P}(H)$ is the set of Borel probability measures on $H$, and $\mathcal{P}_{\mathfrak{w}}(H)$ is the set of measures $\mu \in \mathcal{P}(H)$ such that $\langle\mathfrak{w}, \mu\rangle<\infty$.

\section{Proof of the Main Theorem}

In this section, we state a multiplicative ergodic theorem for the Navier-Stokes system (1.5) and apply it to prove the Main Theorem. Let us start by introducing the following two weight functions

$$
\begin{aligned}
\mathfrak{m}_{\varkappa}(u) & =\exp \left(\varkappa\|u\|^{2}\right), \\
\mathfrak{w}_{m}(u) & =1+\|u\|^{2 m}, \quad u \in H
\end{aligned}
$$


for positive numbers $\varkappa$ and $m$. To avoid triple subscripts, we shall write $C_{\mathfrak{m}}(H)$ and $\mathcal{P}_{\mathfrak{m}}(H)$ instead of $C_{\mathfrak{m}_{\varkappa}}(H)$ and $\mathcal{P}_{\mathfrak{m}_{\varkappa}}(H)$. Recall that the Feynman-Kac semigroup associated with the family $\left(u_{t}, \mathbb{P}_{u}\right)$ is defined by

$$
\mathfrak{P}_{t}^{V} f(u)=\mathbb{E}_{u}\left\{\Xi_{t}^{V} f\left(u_{t}\right)\right\},
$$

where

$$
\Xi_{t}^{V}=\exp \left(\int_{0}^{t} V\left(u_{s}\right) \mathrm{d} s\right)
$$

From estimate (6.21) it follows that, for sufficiently small $\varkappa$ and any $V \in C_{b}(H)$, the application $\mathfrak{P}_{t}^{V}$ maps $C_{\mathfrak{m}}(H)$ into itself. Let $\mathfrak{P}_{t}^{V *}: \mathcal{M}_{+}(H) \rightarrow \mathcal{M}_{+}(H)$ be its dual. Then a measure $\mu \in \mathcal{P}(H)$ and a function $h \in C_{\mathfrak{m}}(H)$ are eigenvectors corresponding to an eigenvalue $\lambda>0$ if

$$
\mathfrak{P}_{t}^{V *} \mu=\lambda^{t} \mu, \quad \mathfrak{P}_{t}^{V} h=\lambda^{t} h \quad \text { for any } t>0 .
$$

We have the following result.

Theorem 2.1. - Under the conditions of the Main Theorem, for any $V \in \mathcal{V}$, there are numbers $m=m(V) \geqslant 1$ and $\gamma_{0}=\gamma_{0}\left(\mathfrak{B}_{0}\right)>0$, where $\mathfrak{B}_{0}=\sum_{j \geqslant 1} b_{j}^{2}$, such that the following assertions hold for any $\varkappa \in\left(0, \gamma_{0}\right)$.

Existence and uniqueness. - There is a unique pair $\left(\mu_{V}, h_{V}\right) \in \mathcal{P}_{\mathfrak{m}}(H) \times C_{\mathfrak{w}}(H)$ of eigenvectors corresponding to an eigenvalue $\lambda_{V}>0$ normalised by the condition $\left\langle h_{V}, \mu_{V}\right\rangle=1$.

Convergence. - For any $f \in C_{\mathfrak{m}}(H), \nu \in \mathcal{P}(H)$, and $R>0$, we have

$$
\begin{aligned}
\lambda_{V}^{-t} \mathfrak{P}_{t}^{V} f \rightarrow\left\langle f, \mu_{V}\right\rangle h_{V} & \text { in } C_{b}\left(B_{H}(R)\right) \cap L^{1}\left(H, \mu_{V}\right) \text { as } t \rightarrow \infty, \\
\lambda_{V}^{-t} \mathfrak{P}_{t}^{V *} \nu & \rightarrow\left\langle h_{V}, \nu\right\rangle \mu_{V} \quad \text { in } \mathcal{M}_{+}(H) \text { as } t \rightarrow \infty .
\end{aligned}
$$

Moreover, for any $M>0$ and $\varkappa^{\prime} \in\left(\varkappa, \gamma_{0}\right)$, the convergence

$$
\lambda_{V}^{-t} \mathbb{E}_{\nu}\left\{f\left(u_{t}\right) \exp \left(\int_{0}^{t} V\left(u_{s}\right) \mathrm{d} s\right)\right\} \rightarrow\left\langle f, \mu_{V}\right\rangle\left\langle h_{V}, \nu\right\rangle \quad \text { as } t \rightarrow \infty
$$

holds uniformly in $\nu \in \Lambda\left(\varkappa^{\prime}, M\right)$.

This theorem is established in Section 5. Here we combine it with some arguments from [JNPS18, MN18a], to prove the Main Theorem.

Proof of the Main Theorem.

Step 1: Reduction. - It suffices to prove the Main Theorem for small $\varkappa$, so we shall assume that $\varkappa \in\left(0, \gamma_{0}\right)$. Let us take any $M>0$ and endow the set

$$
\Theta=\mathbb{R}_{+}^{*} \times \Lambda(\varkappa, M)
$$

with an order relation $\prec$ defined by $\left(t_{1}, \nu_{1}\right) \prec\left(t_{2}, \nu_{2}\right)$ if and only if $t_{1} \leqslant t_{2}$. Then a family $\left\{x_{\theta} \in \mathbb{R}, \theta \in \Theta\right\}$ converges if and only if it converges uniformly with respect to $\nu \in \Lambda(\varkappa, M)$ as $t \rightarrow \infty$. Assume that the following three properties hold.

(1) For any $V \in C_{b}(H)$ and $\nu \in \Lambda(\varkappa, M)$, the following limit exists

$$
Q(V)=\lim _{t \rightarrow \infty} \frac{1}{t} \log \mathbb{E}_{\nu} \exp \left(\int_{0}^{t} V\left(u_{s}\right) \mathrm{d} s\right) .
$$

Moreover, it does not depend and is uniform in $\nu \in \Lambda(\varkappa, M)$. 
(2) There is a vector space $\mathcal{V} \subset C_{b}(H)$ such that its restriction to any compact set $K \subset H$ is dense in $C(K)$, and for any $V \in \mathcal{V}$, there is a unique $\sigma_{V} \in \mathcal{P}(H)$ satisfying the relation

$$
Q(V)=\left\langle V, \sigma_{V}\right\rangle-I\left(\sigma_{V}\right),
$$

where $I(\sigma)$ is the Legendre transform of $Q$ given by (1.8).

(3) There is a function $\Phi: H \rightarrow[0,+\infty]$ with compact level sets $\{u \in H: \Phi(u) \leqslant$ $\alpha$ for any $\alpha \geqslant 0$ such that

$$
\mathbb{E}_{\nu} \exp \left(\int_{0}^{t} \Phi\left(u_{s}\right) \mathrm{d} s\right) \leqslant C e^{c t}, \quad \nu \in \Lambda(\varkappa, M), t>0
$$

for some positive constants $C$ and $c$.

For any $\theta=(t, \nu) \in \Theta$, let us set $r_{\theta}:=t$ and $\zeta_{\theta}:=\zeta_{t}$, where $\zeta_{t}$ is the random probability measure given by $(1.7)$ defined on the probability space $\left(\Omega_{\theta}, \mathcal{F}_{\theta}, \mathbb{P}_{\theta}\right):=\left(\Omega, \mathcal{F}, \mathbb{P}_{\nu}\right)$. The definition of the relation $\prec$ and Properties (1)-(3) imply that the family $\left\{\zeta_{\theta}\right\}$ satisfies the conditions of the Kifer type criterion established in Theorem 3.3 in [JNPS18]. Hence (1.8) defines a good rate function $I$ and for any closed set $F \subset \mathcal{P}(H)$ and open set $G \subset \mathcal{P}(H)$, we have

$$
\begin{gathered}
\limsup _{\theta \in \Theta} \frac{1}{r_{\theta}} \log \mathbb{P}_{\theta}\left\{\zeta_{\theta} \in F\right\} \leqslant-\inf _{\sigma \in F} I(\sigma), \\
\liminf _{\theta \in \Theta} \frac{1}{r_{\theta}} \log \mathbb{P}_{\theta}\left\{\zeta_{\theta} \in G\right\} \geqslant-\inf _{\sigma \in G} I(\sigma) .
\end{gathered}
$$

These two inequalities imply the upper and lower bounds in the Main Theorem, since we have the following equalities

$$
\begin{aligned}
& \limsup _{\theta \in \Theta} \frac{1}{r_{\theta}} \log \mathbb{P}_{\theta}\left\{\zeta_{\theta} \in F\right\}=\limsup _{t \rightarrow \infty} \frac{1}{t} \log \sup _{\nu \in \Lambda} \mathbb{P}_{\nu}\left\{\zeta_{t} \in F\right\}, \\
& \liminf _{\theta \in \Theta} \frac{1}{r_{\theta}} \log \mathbb{P}_{\theta}\left\{\zeta_{\theta} \in G\right\}=\liminf _{t \rightarrow \infty} \frac{1}{t} \log \inf _{\nu \in \Lambda} \mathbb{P}_{\nu}\left\{\zeta_{t} \in G\right\} .
\end{aligned}
$$

Now we turn to the proofs of Properties (1)-(3).

Step 2: Proof of Properties (1)-(3). - Property (3) is the easiest one. It is verified for $\Phi(u)=\varkappa\|u\|_{1}^{2}$ if we choose $\varkappa \in\left(0, \gamma_{0}\right)$. Indeed, (2.8) follows from inequality ${ }^{(4)}(6.20)$, and $\Phi$ has compact level sets, since it is continuous from $H^{1}$ to $\mathbb{R}$ and the embedding $H^{1} \subset H$ is compact.

Properties (1) and (2) are proved using the same methods as in the case of the discrete-time model considered in [JNPS18]. The restriction of $\mathcal{V}$ to any compact set $K \subset H$ is dense in $C(K)$. Taking $f=\mathbf{1}$ in (2.6), we get Property 1 for any $V \in \mathcal{V}$ with $Q(V)=\log \lambda_{V}$. In the case of an arbitrary $V \in C_{b}(H)$, this property is established by using a buc-approximating sequence $V_{n} \in \mathcal{V}$ of $V$ (i.e., $\sup _{n \geqslant 1}\left\|V_{n}\right\|_{\infty}<$ $\infty$ and $\left\|V_{n}-V\right\|_{L^{\infty}(K)} \rightarrow 0$ as $n \rightarrow \infty$ for any compact $K$ in $H$ ) and the exponential tightness of the family $\left\{\zeta_{\theta}\right\}$ (which holds by Property 3 ). The reader is referred to Section 5.6 of [JNPS18] for the details.

${ }^{(4)}$ We shall see in the proof of Theorem 2.1, that $\gamma_{0}$ is the number in Lemma 6.3. 
To prove Property (2), for any $V \in \mathcal{V}$ and $F \in C_{b}(H)$, we consider the following auxiliary Markov semigroup

$$
\mathscr{S}_{t}^{V, F} g(u)=\lambda_{V}^{-t} h_{V}^{-1} \mathfrak{P}_{t}^{V+F}\left(h_{V} g\right)(u), \quad g \in C_{b}(H), t \geqslant 0 .
$$

By Property (1), the following limit exists

$$
Q^{V}(F):=\lim _{t \rightarrow \infty} \frac{1}{t} \log \left(\mathscr{S}_{t}^{V, F} \mathbf{1}\right)(u) .
$$

Let $I^{V}: \mathcal{M}(H) \rightarrow[0,+\infty]$ be the Legendre transform of $Q^{V}$. The arguments of Section 5.7 in [JNPS18] show that $\sigma \in \mathcal{P}(H)$ satisfies (2.7) if and only if $I^{V}(\sigma)=0$. On the other hand, by Proposition 1.3 in [MN18a] (whose proof is the same in our case), the measure $\sigma_{V}=h_{V} \mu_{V}$ is the unique zero of $I^{V}$.

It remains to show that the good rate function $I$ is non-trivial. Assume, by contradiction, that $D_{I}$ is a singleton. Then $I(\mu)=0$ and $I(\sigma)=+\infty$ for $\sigma \in \mathcal{P}(H) \backslash\{\mu\}$, where $\mu$ is the stationary measure of $\left(u_{t}, \mathbb{P}_{u}\right)$. On the other hand, as the Legendre transform is its own inverse, we derive from (1.8) that

$$
Q(V)=\sup _{\sigma \in \mathcal{P}(H)}(\langle V, \sigma\rangle-I(\sigma)) \text { for } V \in C_{b}(H) .
$$

This implies that $Q(V)=\langle V, \mu\rangle$ for any $V \in C_{b}(H)$. Let us take any non-constant $V \in \mathcal{V}$ such that $\langle V, \mu\rangle=0$. Then $Q(V)=0$, and from limit (2.4) with $f=\mathbf{1}$ and $\nu=\mu$ we get $\lambda_{V}=e^{Q(V)}=1$ and

$$
\sup _{t \geqslant 0} \mathbb{E}_{\mu} \exp \left(\int_{0}^{t} V\left(u_{s}\right) \mathrm{d} s\right)<\infty .
$$

Combining the latter with the central limit theorem (see [KS12, Proposition 4.1.4]), we get $V=\mathbf{0}$. This contradicts the assumption that $V$ is non-constant and completes the proof of the Main Theorem.

\section{Checking conditions of Theorem 6.6}

Theorem 2.1 is proved by applying a convergence result for generalised Markov semigroups obtained in [JNPS18, MN18a] and restated here as Theorem 6.6. In this and next sections, we show that the conditions of that theorem are satisfied for the generalised Markov family of transition kernels defined by

$$
P_{t}^{V}(u, \Gamma)=\left(\mathfrak{P}_{t}^{V *} \delta_{u}\right)(\Gamma), \quad \Gamma \in \mathcal{B}(H), u \in H,
$$

if we take $X=H, X_{R}=B_{H^{1}}(R)$, and $\mathfrak{w}=\mathfrak{w}_{m}$ with sufficiently large $m \geqslant 1$.

\subsection{Growth estimates}

Estimate (6.24) implies that the measure $P_{t}^{V}(u, \cdot)$ is concentrated on the space $H^{1}=\bigcup_{R=1}^{\infty} X_{R}=X_{\infty}$ for any $V \in C_{b}(H), t>0$, and $u \in H$. The boundedness of $V$ implies that $\sup _{t \in[0,1]}\left\|\mathfrak{P}_{t}^{V} \mathbf{1}\right\|_{\infty}<\infty$. So the following proposition gives the growth condition in Theorem 6.6. 
Proposition 3.1. - For any $V \in C_{b}(H)$, there are positive numbers $m$ and $R_{0}$ such that

$$
\sup _{t \geqslant 0} \frac{\left\|\mathfrak{P}_{t}^{V} \mathfrak{w}\right\|_{L_{\mathfrak{w}}^{\infty}}}{\left\|\mathfrak{P}_{t}^{V} \mathbf{1}\right\|_{R_{0}}}<\infty
$$

where $\mathfrak{w}=\mathfrak{w}_{m}$ and $\|\cdot\|_{R_{0}}$ is the $L^{\infty}$ norm on $X_{R_{0}}$.

Proof. - Replacing $V$ by $V-\inf _{H} V$, we can assume that $V$ is non-negative.

Step 1. - Let us show that there are integers $m, R_{0} \geqslant 1$ such that

$$
\sup _{t \geqslant 0} \frac{\left\|\mathfrak{P}_{t}^{V} \mathbf{1}\right\|_{L_{\mathfrak{w}}^{\infty}}}{\left\|\mathfrak{P}_{t}^{V} \mathbf{1}\right\|_{R_{0}}}<\infty
$$

Indeed, let $\tau(R)$ be the first hitting time of the set $X_{R}$ defined by (6.26), and let $m$ and $R_{0}$ be the integers in Proposition 6.4 for $\gamma=\|V\|_{\infty}$. Then for any $u \in H$, we have

$$
\mathfrak{P}_{t}^{V} \mathbf{1}(u)=\mathbb{E}_{u} \Xi_{t}^{V}=\mathbb{E}_{u}\left\{\mathbb{I}_{G_{t}} \Xi_{t}^{V}\right\}+\mathbb{E}_{u}\left\{\mathbb{I}_{G_{t}^{c}} \Xi_{t}^{V}\right\}=: I_{1}+I_{2},
$$

where $\Xi_{t}^{V}$ is given by (2.3) and $G_{t}=\left\{\tau\left(R_{0}\right)>t\right\}$. As $V$ is non-negative, we have $\mathfrak{P}_{t}^{V} \mathbf{1}(u) \geqslant 1$. This and (6.27) imply that

$$
I_{1} \leqslant \mathbb{E}_{u} \Xi_{\tau\left(R_{0}\right)}^{V} \leqslant \mathbb{E}_{u} \exp \left(\gamma \tau\left(R_{0}\right)\right) \leqslant C \mathfrak{w}(u) \leqslant C \mathfrak{w}(u)\left\|\mathfrak{P}_{t}^{V} \mathbf{1}\right\|_{R_{0}}
$$

By the strong Markov property and (6.27),

$$
\begin{aligned}
I_{2} & \leqslant \mathbb{E}_{u}\left\{\mathbb{I}_{G_{t}} \Xi_{\tau\left(R_{0}\right)}^{V} \mathbb{E}_{u\left(\tau\left(R_{0}\right)\right)} \Xi_{t}^{V}\right\} \\
& \leqslant \mathbb{E}_{u}\left\{e^{\gamma \tau\left(R_{0}\right)}\right\}\left\|\mathfrak{P}_{t}^{V} \mathbf{1}\right\|_{R_{0}} \leqslant C \mathfrak{w}(u)\left\|\mathfrak{P}_{t}^{V} \mathbf{1}\right\|_{R_{0}} .
\end{aligned}
$$

Inequalities (3.3)-(3.5) imply (3.2).

Step 2. - It suffices to prove (3.1) for integer times $k \geqslant 1$ :

$$
\sup _{k \geqslant 0} \frac{\left\|\mathfrak{P}_{k}^{V} \mathfrak{w}\right\|_{L_{\mathfrak{w}}^{\infty}}}{\left\|\mathfrak{P}_{k}^{V} \mathbf{1}\right\|_{R_{0}}}<\infty
$$

Indeed, the semigroup property and the fact that $V$ is non-negative and bounded imply that

$$
\begin{aligned}
\left\|\mathfrak{P}_{t}^{V} \mathfrak{w}\right\|_{L_{\mathfrak{w}}^{\infty}} & =\left\|\mathfrak{P}_{t-[t]}^{V}\left(\mathfrak{P}_{[t]}^{V} \mathfrak{w}\right)\right\|_{L_{\mathfrak{w}}^{\infty}} \leqslant C_{0}\left\|\mathfrak{P}_{[t]}^{V} \mathfrak{w}\right\|_{L_{\mathfrak{w}}^{\infty}}, \\
\left\|\mathfrak{P}_{t}^{V} \mathbf{1}\right\|_{R_{0}} & \geqslant\left\|\mathfrak{P}_{[t]}^{V} \mathbf{1}\right\|_{R_{0}},
\end{aligned}
$$

where $[t]$ is the integer part of $t$ and $C_{0}:=\sup _{s \in[0,1]}\left\|\mathfrak{P}_{s}^{V} \mathfrak{w}\right\|_{L_{\mathfrak{w}}^{\infty}}$. By (6.23), we have

$$
C_{0} \leqslant e^{\gamma} \sup _{s \in[0,1]}\left\|\mathfrak{P}_{s} \mathfrak{w}\right\|_{L_{\mathfrak{w}}^{\infty}}<\infty
$$

where $\mathfrak{P}_{t}=\mathfrak{P}_{t}^{0}$ is the Markov operator associated with (1.5). 
Step 3. - To prove (3.6), we use the Markov property and (6.23):

$$
\begin{aligned}
\mathfrak{P}_{k}^{V} \mathfrak{w}(u) & \leqslant e^{\gamma} \mathbb{E}_{u}\left\{\Xi_{k-1}^{V} \mathfrak{w}\left(u_{k}\right)\right\} \\
& =e^{\gamma} \mathbb{E}_{u}\left\{\Xi_{k-1}^{V} \mathbb{E}_{u_{k-1}} \mathfrak{w}\left(u_{1}\right)\right\} \\
& \leqslant e^{\gamma} \mathbb{E}_{u}\left\{\Xi_{k-1}^{V}\left[e^{-m \alpha_{1}} \mathfrak{w}\left(u_{k-1}\right)+C_{1}\right]\right\} \\
& \leqslant q \mathfrak{P}_{k-1}^{V} \mathfrak{w}(u)+e^{\gamma} C_{1} \mathfrak{P}_{k-1}^{V} \mathbf{1}(u),
\end{aligned}
$$

where we choose $m>\gamma / \alpha_{1}$, so that $q:=e^{\gamma-m \alpha_{1}}<1$. Iterating this inequality and using the fact that the sequence $\left\{\left\|\mathfrak{P}_{k}^{V} \mathbf{1}\right\|_{R_{0}}\right\}$ is a non-decreasing in $k$, we obtain

$$
\mathfrak{P}_{k}^{V} \mathfrak{w}(u) \leqslant q^{k} \mathfrak{w}(u)+(1-q)^{-1} e^{\gamma} C_{1} \mathfrak{P}_{k}^{V} \mathbf{1}(u) .
$$

This and (3.2) imply (3.6).

We shall also need the following growth estimates with two other weights.

Proposition 3.2. - Let $V \in C_{b}(H)$ and let $R_{0}$ and $\gamma_{0}$ be the numbers in Proposition 3.1 and Lemma 6.3, respectively. Then for any $\varkappa \in\left(0, \gamma_{0}\right)$, we have

$$
\begin{aligned}
& \sup _{t \geqslant 0} \frac{\left\|\mathfrak{P}_{t}^{V} \mathfrak{m}\right\|_{L_{\mathfrak{m}}^{\infty}}}{\left\|\mathfrak{P}_{t}^{V} \mathbf{1}\right\|_{R_{0}}}<\infty, \\
& \sup _{t \geqslant 1} \frac{\left\|\mathfrak{P}_{t}^{V} F\right\|_{L_{\mathfrak{m}}^{\infty}}}{\left\|\mathfrak{P}_{t}^{V} \mathbf{1}\right\|_{R_{0}}}<\infty
\end{aligned}
$$

where $\mathfrak{m}=\mathfrak{m}_{\varkappa}$ and $F(u)=\|u\|_{1}^{2}$.

Proof.

Step 1: Proof of (3.7). - As in the previous proof, we can assume that $V$ is non-negative and $t=k$ is integer. We take any $A>0$ and write

$$
\begin{aligned}
\mathfrak{P}_{k}^{V} \mathfrak{m}(u) & =\mathbb{E}_{u}\left\{\mathbb{I}_{\left\{\left\|u_{k}\right\|^{2} \leqslant A\right\}} \Xi_{k}^{V} \mathfrak{m}\left(u_{k}\right)\right\}+\mathbb{E}_{u}\left\{\mathbb{I}_{\left\{\left\|u_{k}\right\|^{2}>A\right\}} \Xi_{k}^{V} \mathfrak{m}\left(u_{k}\right)\right\} \\
& =: I_{k}+J_{k} .
\end{aligned}
$$

By (3.2), we have

$$
\left\|\mathfrak{P}_{k}^{V} \mathbf{1}\right\|_{L_{\mathrm{m}}^{\infty}} \leqslant C_{2}\left\|\mathfrak{P}_{k}^{V} \mathbf{1}\right\|_{R_{0}}
$$

hence

$$
\left\|I_{k}\right\|_{L_{\mathbf{m}}^{\infty}} \leqslant e^{\varkappa A}\left\|\mathfrak{P}_{k}^{V} \mathbf{1}\right\|_{L_{\mathfrak{m}}^{\infty}} \leqslant C_{2} e^{\varkappa A}\left\|\mathfrak{P}_{k}^{V} \mathbf{1}\right\|_{R_{0}} .
$$

To estimate $J_{k}$, we use the Markov property and (6.22)

$$
\begin{aligned}
J_{k}(u) & \leqslant A^{-1} \mathbb{E}_{u}\left\{\left\|u_{k}\right\|^{2} \Xi_{k}^{V} \mathfrak{m}\left(u_{k}\right)\right\} \leqslant A^{-1} e^{\gamma} \mathbb{E}_{u}\left\{\left\|u_{k}\right\|^{2} \Xi_{k-1}^{V} \mathfrak{m}\left(u_{k}\right)\right\} \\
& =A^{-1} e^{\gamma} \mathbb{E}_{u}\left\{\Xi_{k-1}^{V} \mathbb{E}_{u_{k-1}}\left\{\left\|u_{1}\right\|^{2} \mathfrak{m}\left(u_{1}\right)\right\}\right\} \leqslant A^{-1} C_{3} \mathfrak{P}_{k-1}^{V} \mathfrak{m}(u) .
\end{aligned}
$$

Combining this with (3.9) and (3.10), and choosing $A$ so large that $q:=A^{-1} C_{3}<1$, we get

$$
\left\|\mathfrak{P}_{k}^{V} \mathfrak{m}\right\|_{L_{\mathfrak{m}}^{\infty}} \leqslant C_{2} e^{\varkappa A}\left\|\mathfrak{P}_{k}^{V} \mathbf{1}\right\|_{R_{0}}+q\left\|\mathfrak{P}_{k-1}^{V} \mathfrak{m}\right\|_{L_{\mathfrak{m}}^{\infty}}
$$

Iterating, we obtain

$$
\left\|\mathfrak{P}_{k}^{V} \mathfrak{m}\right\|_{L_{\mathfrak{m}}^{\infty}} \leqslant C_{2} e^{\varkappa A}(1-q)^{-1}\left\|\mathfrak{P}_{k}^{V} \mathbf{1}\right\|_{R_{0}}+q^{k} .
$$


As $\mathfrak{P}_{k}^{V} \mathbf{1}(u) \geqslant 1$, we arrive at the required inequality (3.7).

Step 2: Proof of (3.8). - For any $t \geqslant 1$, we have

$$
\mathfrak{P}_{t}^{V} F=\mathfrak{P}_{t-1}^{V}\left(\mathfrak{P}_{1}^{V} F\right) \leqslant e^{\gamma} \mathfrak{P}_{t-1}^{V}\left(\mathfrak{P}_{1} F\right) .
$$

So (6.24) and (3.7) imply that

$$
\mathfrak{P}_{t}^{V} F(u) \leqslant C_{4} \mathfrak{P}_{t-1}^{V} \mathfrak{w}_{8}(u) \leqslant C_{5} \mathfrak{P}_{t-1}^{V} \mathfrak{m}(u) \leqslant C_{6}\left\|\mathfrak{P}_{t}^{V} \mathbf{1}\right\|_{R_{0}} \mathfrak{m}(u)
$$

This proves (3.8).

\subsection{Time-continuity}

The following lemma proves the time-continuity property.

Lemma 3.3. - The function $t \mapsto \mathfrak{P}_{t}^{V} g(u)$ is continuous from $\mathbb{R}_{+}$to $\mathbb{R}$ for any $V \in C_{b}(H), g \in C_{\mathfrak{w}}(H), u \in H$, and $\mathfrak{w}=\mathfrak{w}_{m}$ with any $m \geqslant 1$.

Proof. - Let us show the continuity at the point $T \geqslant 0$. For any $t \geqslant 0$, we write

$$
\begin{aligned}
\mathfrak{P}_{T}^{V} g(u)-\mathfrak{P}_{t}^{V} g(u) & =\mathbb{E}_{u}\left\{\left[\Xi_{T}^{V}-\Xi_{t}^{V}\right] g\left(u_{t}\right)\right\}+\mathbb{E}_{u}\left\{\left[g\left(u_{T}\right)-g\left(u_{t}\right)\right] \Xi_{T}^{V}\right\} \\
& =: S_{1}+S_{2} .
\end{aligned}
$$

As $V$ is bounded and $g \in C_{\mathfrak{w}}(H)$, we have

$$
\begin{aligned}
\left|S_{1}\right| & \leqslant \mathbb{E}_{u}\left\{\left|\exp \left(\int_{t}^{T} V\left(u_{s}\right) \mathrm{d} s\right)-1\right| \Xi_{t}^{V}\left|g\left(u_{t}\right)\right|\right\} \\
& \leqslant\|g\|_{L_{\mathfrak{w}}^{\infty}}\left(e^{|T-t|\|V\|_{\infty}}-1\right) e^{T\|V\|_{\infty}} \mathbb{E}_{u} \mathfrak{w}\left(u_{t}\right) .
\end{aligned}
$$

Combining this with (6.23), we get $S_{1} \rightarrow 0$ as $t \rightarrow T$. To estimate $S_{2}$, we take any $R>0$ and write

$$
\begin{aligned}
e^{-T\|V\|_{\infty}\left|S_{2}\right|} & \leqslant \mathbb{E}_{u}\left|g\left(u_{T}\right)-g\left(u_{t}\right)\right| \\
& =\mathbb{E}_{u}\left\{\mathbb{I}_{G_{R}^{c}}\left|g\left(u_{T}\right)-g\left(u_{t}\right)\right|\right\}+\mathbb{E}_{u}\left\{\mathbb{I}_{G_{R}}\left|g\left(u_{T}\right)-g\left(u_{t}\right)\right|\right\} \\
& =: S_{3}+S_{4},
\end{aligned}
$$

where $G_{R}:=\left\{u_{t}, u_{T} \in B_{H}(R)\right\}$. From $g \in C_{\mathfrak{w}}(H)$ and (6.23) we derive

$$
\begin{aligned}
S_{3} & \leqslant C_{1} \mathbb{E}_{u}\left\{\mathbb{I}_{G_{R}^{c}}\left(\mathfrak{w}\left(u_{T}\right)+\mathfrak{w}\left(u_{t}\right)\right)\right\} \\
& \leqslant C_{1} R^{-1} \mathbb{E}_{u}\left\{\mathfrak{w}^{2}\left(u_{T}\right)+\mathfrak{w}^{2}\left(u_{t}\right)\right\} \leqslant C_{2} R^{-1} \mathfrak{w}^{2}(u) .
\end{aligned}
$$

On the other hand, by the Lebesgue theorem on dominated convergence, for any $R>0$, we have $S_{4} \rightarrow 0$ as $t \rightarrow T$. Choosing $R>0$ sufficiently large and $t$ sufficiently close to $T$, we see that $S_{3}+S_{4}$ can be made arbitrarily small. This shows that $S_{2} \rightarrow 0$ as $t \rightarrow T$ and completes the proof of the lemma. 


\subsection{Uniform irreducibility}

As $V$ is a bounded function, we have

$$
P_{t}^{V}(u, \mathrm{~d} v) \geqslant e^{-t\|V\|_{\infty}} P_{t}(u, \mathrm{~d} v), \quad u \in H,
$$

where $P_{t}(u, \cdot)$ is the transition function of the Markov family $\left(u_{t}, \mathbb{P}_{u}\right)$. Thus to show the uniform irreducibility of $\left\{P_{t}^{V}\right\}$, it suffices to prove the following result.

Proposition 3.4. - The family $\left\{P_{t}\right\}$ is uniformly irreducible with respect to the sequence $\left\{X_{R}\right\}$, i.e., for any integers $\rho, R \geqslant 1$ and any $r>0$, there are positive numbers $l=l(\rho, r, R)$ and $p=p(\rho, r)$ such that

$$
P_{l}\left(u, B_{H}(\hat{u}, r)\right) \geqslant p, \quad u \in B_{H}(R), \hat{u} \in X_{\rho} .
$$

Proof.

Step 1. - There is a number $d>0$ such that for any $R \geqslant 1$, we have

$$
P_{t}\left(u, X_{d}\right) \geqslant \frac{1}{2}, \quad u \in B_{H}(R)
$$

for sufficiently large $t=t(R)$. Indeed, combining (6.23), (6.24), and the Markov property, we get

$$
\mathbb{E}_{u}\left\|u_{t}\right\|_{1}^{2} \leqslant C\left(e^{-8 \alpha_{1} t} R^{8}+1\right), \quad u \in B_{H}(R), t \geqslant 1 .
$$

Taking $t$ so large that $e^{-8 \alpha_{1} t} R^{8}<1$ and $d>2 \sqrt{C}$ and using the Chebyshev inequality, we arrive at

$$
P_{t}\left(u, X_{d}\right) \geqslant 1-d^{-2} C\left(e^{-8 \alpha_{1} t} R^{8}+1\right) \geqslant \frac{1}{2} .
$$

Step 2. - By Lemma 3.3.11 in [KS12], for any non-degenerate ball $B \subset H$, there is $p_{1}=p_{1}(d, B)>0$ such that

$$
P_{1}(u, B) \geqslant p_{1}, \quad u \in X_{d} .
$$

Combining this with a simple compactness and continuity argument, we get

$$
P_{1}\left(u, B_{H}(\hat{u}, r)\right) \geqslant p_{2}, \quad u \in X_{d}, \hat{u} \in X_{\rho}
$$

for some $p_{2}=p_{2}(d, \rho, r)>0$. This estimate, (3.12), and the Kolmogorov-Chapman relation imply (3.11) with $l=t+1$ and $p=p_{2} / 2$.

\subsection{Existence of an eigenvector}

Here we show that the dual operator $\mathfrak{P}_{t}^{V *}$ has an eigenvector and give some decay estimates for it.

Proposition 3.5. - For any $V \in C_{b}(H)$ and $t>0$, the operator $\mathfrak{P}_{t}^{V *}$ has at least one eigenvector $\mu_{t, V} \in \mathcal{P}(H)$ with a positive eigenvalue $\lambda_{t, V}$ :

$$
\mathfrak{P}_{t}^{V *} \mu_{t, V}=\lambda_{t, V} \mu_{t, V} .
$$


Moreover, any such eigenvector satisfies

$$
\begin{aligned}
& \int_{H}\left(\|u\|_{1}^{n}+\mathfrak{m}_{\varkappa}(u)\right) \mu_{t, V}(\mathrm{~d} u)<\infty, \\
& \left\|\mathfrak{P}_{t}^{V} \mathfrak{w}_{m}\right\|_{X_{R}} \int_{X_{R}^{c}} \mathfrak{w}_{m}(u) \mu_{t, V}(\mathrm{~d} u) \rightarrow 0 \quad \text { as } R \rightarrow \infty
\end{aligned}
$$

for any $\varkappa \in\left(0, \gamma_{0}\right)$ and $n, m \geqslant 1$.

\section{Proof.}

Step 1: Estimate (3.14). - Let us fix $t>0$, and let $\mu \in \mathcal{P}(H)$ be an eigenvector of the operator $\mathfrak{P}_{t}^{V *}$ corresponding to an eigenvalue $\lambda>0$. Let us show that $\mu \in \mathcal{P}_{\mathfrak{m}}(H)$ with $\mathfrak{m}=\mathfrak{m}_{\varkappa}$ for any $\varkappa \in\left(0, \gamma_{0}\right)$. Indeed, for any measurable function $f: H \rightarrow$ $\mathbb{R}_{+} \cup\{+\infty\}$, we have

$$
\langle f, \mu\rangle=\lambda^{-1}\left\langle\mathfrak{P}_{t}^{V} f, \mu\right\rangle \leqslant \lambda^{-1} e^{t\|V\|_{\infty}}\left\langle\mathfrak{P}_{t} f, \mu\right\rangle .
$$

Taking $f=\mathfrak{m}_{\varkappa}$, any number $A>0$, and setting ${ }^{(5)} C_{1}=\lambda^{-1} e^{t\|V\|_{\infty}}$, we obtain

$$
\begin{aligned}
\int_{H} \mathfrak{m}_{\varkappa}(u) \mu(\mathrm{d} u) & \leqslant C_{1} \int_{H} \mathbb{E}_{u}\left\{\mathfrak{m}_{\varkappa}\left(u_{t}\right)\right\} \mu(\mathrm{d} u) \\
& =C_{1} \int_{H}\left(\mathbb{E}_{u}\left\{\mathbb{I}_{\left\{\left\|u_{t}\right\|^{2} \leqslant A\right\}} \mathfrak{m}_{\varkappa}\left(u_{t}\right)\right\}+\mathbb{E}_{u}\left\{\mathbb{I}_{\left\{\left\|u_{t}\right\|^{2}>A\right\}} \mathfrak{m}_{\varkappa}\left(u_{t}\right)\right\}\right) \mu(\mathrm{d} u) \\
& \leqslant C_{1} \int_{H}\left(\exp (\varkappa A)+A^{-1} \mathbb{E}_{u}\left\{\left\|u_{t}\right\|^{2} \mathfrak{m}_{\varkappa}\left(u_{t}\right)\right\}\right) \mu(\mathrm{d} u) \\
& \leqslant C_{1} \int_{H}\left(\exp (\varkappa A)+C_{2} A^{-1} \mathfrak{m}_{\varkappa}(u)\right) \mu(\mathrm{d} u),
\end{aligned}
$$

where we used inequality (6.22). Choosing $A>C_{1} C_{2}$, we get

$$
\int_{H} \mathfrak{m}_{\varkappa}(u) \mu(\mathrm{d} u) \leqslant C_{1}\left(1-C_{1} C_{2} A^{-1}\right)^{-1} \exp (\varkappa A)<\infty,
$$

so ${ }^{(6)} \mu \in \mathcal{P}_{\mathfrak{m}}(H)$. Taking $f(u)=\|u\|_{1}^{n}$ in (3.16) and using (6.24) and (3.17), we obtain

$$
\int_{H}\|u\|_{1}^{n} \mu(\mathrm{d} u) \leqslant C_{1} \int_{H} \mathbb{E}_{u}\left\{\left\|u_{t}\right\|_{1}^{n}\right\} \mu(\mathrm{d} u) \leqslant C_{3} \int_{H}\left(1+\|u\|^{8 n}\right) \mu(\mathrm{d} u)<\infty
$$

for any $n \geqslant 1$. This proves (3.14).

Step 2: Limit (3.15). — From (6.23) it follows that

$$
\begin{aligned}
\left\|\mathfrak{P}_{t}^{V} \mathfrak{w}_{m}\right\|_{X_{R}} & \leqslant e^{t\|V\|_{\infty}} \sup _{u \in X_{R}} \mathbb{E}_{u} \mathfrak{w}_{m}\left(u_{t}\right) \\
& \leqslant C_{4} \sup _{u \in B_{H}(R)} \mathfrak{w}_{m}(u)=C_{4}\left(1+R^{2 m}\right) .
\end{aligned}
$$

Using the Cauchy-Schwarz inequality, (3.14), and the Chebyshev inequality, we see that

$$
\int_{X_{R}^{c}} \mathfrak{w}_{m}(u) \mu(\mathrm{d} u) \leqslant\left\langle\mathfrak{w}_{m}^{2}, \mu\right\rangle^{1 / 2} \mu\left(X_{R}^{c}\right)^{1 / 2} \leqslant C_{5} R^{-n} .
$$

Combining this with (3.18) and choosing $n>2 m$, we obtain (3.15).

${ }^{(5)}$ We do not indicate the dependence of different constants on $V, t, m, n$, and $\varkappa$.

${ }^{(6)}$ Note that this proof is formal. A rigorous proof can be obtained by applying the above arguments to bounded approximations of $\mathfrak{m}$. 
Step 3: Construction of an eigenvector. - Let us take any $A>0$ and $m \geqslant 1$ and define the convex set

$$
D_{A, m}:=\left\{\nu \in \mathcal{P}(H):\left\langle\mathfrak{w}_{m}, \nu\right\rangle \leqslant A\right\} .
$$

By the Fatou lemma, $D_{A, m}$ is closed in $\mathcal{P}(H)$. Consider the continuous mapping

$$
G:=G(t, V): D_{A, m} \rightarrow \mathcal{P}(H), \quad \nu \mapsto \frac{\mathfrak{P}_{t}^{V *} \nu}{\mathfrak{P}_{t}^{V *} \nu(H)}
$$

Let us show that $G\left(D_{A, m}\right) \subset D_{A, m}$ for an appropriate choice of $A$ and $m$, and that $G\left(D_{A, m}\right)$ is compact in $\mathcal{P}(H)$. In view of the Leray-Schauder theorem, this will imply the existence of an eigenvector $\mu \in D_{A, m}$ satisfying (3.13) with eigenvalue $\lambda=\mathfrak{P}_{t}^{V *} \mu(H)>0$. From (6.23) we derive that

$$
\begin{aligned}
\left\langle\mathfrak{w}_{m}, G(\nu)\right\rangle & \leqslant \exp \{t \operatorname{Osc}(V)\}\left\langle\mathfrak{w}_{m}, \mathfrak{P}_{t}^{*} \nu\right\rangle \\
& \leqslant \exp \left\{t\left(\operatorname{Osc}(V)-m \alpha_{1}\right)\right\}\left\langle\mathfrak{w}_{m}, \nu\right\rangle+C_{6},
\end{aligned}
$$

where $\operatorname{Osc}(V):=\sup _{u \in H} V(u)-\inf _{u \in H} V(u)$ is the oscillation of $V$. Choosing $A$ and $m$ so large that $\exp \left\{t\left(\operatorname{Osc}(V)-m \alpha_{1}\right)\right\} \leqslant 1 / 2$ and $A \geqslant 2 C_{6}$, we get that $G\left(D_{A, m}\right) \subset D_{A, m}$. In view of the Prokhorov compactness criterion (see [Dud02, Theorem 11.5.4]), to prove that $G\left(D_{A, m}\right)$ is relatively compact, it suffices to check that

$$
\int_{H}\|u\|_{1}^{2} \mathfrak{P}_{t}^{V *} \nu(\mathrm{d} u) \leqslant C_{7} \quad \text { for any } \nu \in D_{A, m} .
$$

Using (6.24) and the fact that $V$ is bounded, we get

$$
\begin{aligned}
\int_{H}\|u\|_{1}^{2} \mathfrak{P}_{t}^{V *} \nu(\mathrm{d} u) & \leqslant \exp \left(t\|V\|_{\infty}\right) \int_{H}\|u\|_{1}^{2}\left(\mathfrak{P}_{t}^{*} \nu\right)(\mathrm{d} u) \\
& \leqslant C_{8} \int_{H}\|u\|^{8} \nu(\mathrm{d} u) \\
& \leqslant C_{9} \int_{H} \mathfrak{w}_{m}(u) \nu(\mathrm{d} u) \leqslant C_{9} A=: C_{7} .
\end{aligned}
$$

Thus there is an eigenvector $\mu \in D_{A, m}$.

\section{Uniform Feller property}

In this section, we establish the following result.

Theorem 4.1. - For any $V \in \mathcal{V}$, the family $\left\{P_{t}^{V}\right\}$ satisfies the uniform Feller property with respect to the sequence $\left\{X_{R}\right\}$, i.e., there is an integer $R_{0} \geqslant 1$ such that the family $\left\{\left\|\mathfrak{P}_{t}^{V} \mathbf{1}\right\|_{R}^{-1} \mathfrak{P}_{t}^{V} \psi, t \geqslant 0\right\}$ is uniformly equicontinuous on $X_{R}$ for any $\psi \in \mathcal{V}$ and $R \geqslant R_{0}$.

See the papers [JNPS15, JNPS18, MN18b] for similar results in the case of a discrete-time random dynamical system and [MN18a] for the case of the stochastic damped nonlinear wave equation. The main difficulty in the proof of Theorem 4.1 comes from the fact that the oscillation of the potential $V$ can be arbitrarily large. To overcome this, we introduce a new auxiliary equation in the construction of the coupling processes and choose carefully the parameters in order to have a stabilisation property with an appropriate rate. 


\subsection{Construction of coupling processes}

The coupling processes are constructed following the arguments of [MN18a]. Let us take any $z, z^{\prime} \in H$ and denote by $u_{t}$ and $u_{t}^{\prime}$ the solutions of (1.5) issued from $z$ and $z^{\prime}$. For any integer $N \geqslant 1$ and number $\lambda>0$, let $v$ be the solution of the following problem

$$
\dot{v}+B(v)+L v+\mathrm{P}_{N}[\lambda(v-u)+B(u)-B(v)]=h+\eta(t), \quad v(0)=z^{\prime},
$$

where $\eta$ is defined by (1.3). We denote by $\nu\left(z, z^{\prime}\right)$ and $\nu^{\prime}\left(z^{\prime}\right)$ the laws of processes $\{v(t), t \in J\}$ and $\left\{u^{\prime}(t), t \in J\right\}$, respectively, where $J=[0,1]$. We shall use the following result.

Proposition 4.2. - There exists an integer $N_{1} \geqslant 1$ such that if $N \geqslant N_{1}$ and $\lambda \geqslant N^{2} / 2$, then for any $\varepsilon>0$ and $z, z^{\prime} \in H$, we have

$$
\left\|\nu\left(z, z^{\prime}\right)-\nu^{\prime}\left(z^{\prime}\right)\right\|_{v a r} \leqslant \varepsilon^{a}+2\left[\exp \left(C_{\lambda, N} \varepsilon^{a-2}\left\|z-z^{\prime}\right\|^{2} e^{C\left(\|z\|^{2}+\left\|z^{\prime}\right\|^{2}\right)}\right)-1\right]^{1 / 2}
$$

where $\|\cdot\|_{\text {var }}$ denotes the total variation distance on $\mathcal{P}(C(J ; H))$ and $a<2, C$, and $C_{\lambda, N}$ are positive constants not depending on $\varepsilon, z, z^{\prime}$.

See Section 6.2 for the proof. By Proposition 1.2.28 in [KS12], there is a probability space $(\hat{\Omega}, \hat{\mathcal{F}}, \hat{\mathbb{P}})$ and measurable functions $\mathcal{Z}, \mathcal{Z}^{\prime}: H \times H \times \hat{\Omega} \rightarrow C(J ; H)$ such that $\left(\mathcal{Z}\left(z, z^{\prime}\right), \mathcal{Z}^{\prime}\left(z, z^{\prime}\right)\right)$ is a maximal coupling for $\left(\nu\left(z, z^{\prime}\right), \nu\left(z^{\prime}\right)\right)$ for any $z, z^{\prime} \in H$. We denote by $\tilde{v}$ and $\tilde{u}_{t}^{\prime}$ the restrictions of $\mathcal{Z}$ and $\mathcal{Z}^{\prime}$ to time $t \in J$. Then $\tilde{v}_{t}$ is a solution of

$$
\dot{\tilde{v}}+B(\tilde{v})+L \tilde{v}+\mathrm{P}_{N}[\lambda \tilde{v}-B(\tilde{v})]=h+\psi(t), \quad \tilde{v}(0)=z^{\prime},
$$

where the process $\left\{\int_{0}^{t} \psi(s) \mathrm{d} s, t \in J\right\}$ has the same law as

$$
\left\{W(t)-\int_{0}^{t} \mathrm{P}_{N}\left[B\left(u_{s}\right)-\lambda u_{s}\right] \mathrm{d} s, t \in J\right\} .
$$

Let $\tilde{u}_{t}$ be a solution of

$$
\dot{\tilde{u}}+B(\tilde{u})+L \tilde{u}+\mathrm{P}_{N}[\lambda \tilde{u}-B(\tilde{u})]=h+\psi(t), \quad \tilde{u}(0)=z .
$$

Then $\left\{\tilde{u}_{t}, t \in J\right\}$ has the same law as $\left\{u_{t}, t \in J\right\}$. Now the coupling operators $\mathcal{R}$ and $\mathcal{R}^{\prime}$ are defined by

$$
\mathcal{R}_{t}\left(z, z^{\prime}, \omega\right)=\tilde{u}_{t}, \quad \mathcal{R}_{t}^{\prime}\left(z, z^{\prime}, \omega\right)=\tilde{u}_{t}^{\prime}, \quad z, z^{\prime} \in H, \omega \in \hat{\Omega}, t \in J .
$$

By Proposition 4.2 , for any $\varepsilon>0, N \geqslant N_{1}$, and $\lambda \geqslant N^{2} / 2$, we have

$$
\begin{aligned}
\hat{\mathbb{P}}\left\{\exists t \in J \text { s.t. } \tilde{v}_{t} \neq \tilde{u}_{t}^{\prime}\right\} & \\
& \leqslant \varepsilon^{a}+2\left[\exp \left(C_{\lambda, N} \varepsilon^{a-2}\left\|z-z^{\prime}\right\|^{2} e^{C\left(\|z\|^{2}+\left\|z^{\prime}\right\|^{2}\right)}\right)-1\right]^{1 / 2} .
\end{aligned}
$$

Let $\left(\Omega^{k}, \mathcal{F}^{k}, \mathbb{P}^{k}\right), k \geqslant 0$ be a sequence of independent copies of $(\hat{\Omega}, \hat{\mathcal{F}}, \hat{\mathbb{P}})$ and $(\Omega, \mathcal{F}, \mathbb{P})$ the direct product of $\left(\Omega^{k}, \mathcal{F}^{k}, \mathbb{P}^{k}\right)$. For any $\omega=\left(\omega^{1}, \omega^{2}, \ldots\right) \in \Omega$ and $z, z^{\prime} \in H$, we set $\tilde{u}_{0}=z, \tilde{u}_{0}^{\prime}=z^{\prime}$, and

$$
\begin{aligned}
& \tilde{u}_{t}(\omega)=\mathcal{R}_{s}\left(\tilde{u}_{k}(\omega), \tilde{u}_{k}^{\prime}(\omega), \omega^{k}\right), \quad \tilde{u}_{t}^{\prime}(\omega)=\mathcal{R}_{s}^{\prime}\left(\tilde{u}_{k}(\omega), \tilde{u}_{k}^{\prime}(\omega), \omega^{k}\right), \\
& \tilde{v}_{t}(\omega)=\mathcal{Z}_{s}\left(\tilde{u}_{k}(\omega), \tilde{u}_{k}^{\prime}(\omega), \omega^{k}\right) \text {, }
\end{aligned}
$$


where $t=s+k, s \in[0,1)$. We shall say that $\left(\tilde{u}_{t}, \tilde{u}_{t}^{\prime}\right)$ is a coupled trajectory at level $(N, \lambda)$ issued from $\left(z, z^{\prime}\right)$.

\subsection{Proof of Theorem 4.1}

Step 1: Stratification. - Let us take any functions $V, \psi \in \mathcal{V}$ and points $z, z^{\prime} \in X_{R}$ such that $d:=\left\|z-z^{\prime}\right\| \leqslant 1$. We need to prove the uniform equicontinuity of the family $\left\{g_{t}, t \geqslant 0\right\}$ on $X_{R}$, where

$$
g_{t}=\left\|\mathfrak{P}_{t}^{V} \mathbf{1}\right\|_{R}^{-1} \mathfrak{P}_{t}^{V} \psi
$$

Without loss of generality, we can assume that $\psi$ and $V$ are non-negative, $\psi \leqslant 1$, and the integer $N$ in representation (1.10) is the same for $\psi$ and $V$ (we denote it by $N_{0}$ ). Let $\left(u_{t}, u_{t}^{\prime}\right):=\left(\tilde{u}_{t}, \tilde{u}_{t}^{\prime}\right)$ be a coupled trajectory at level $(N, \lambda)$ issued from $\left(z, z^{\prime}\right)$ and let $v_{t}:=\tilde{v}_{t}$ be the associated process. The parameters $N \geqslant N_{0}$ and $\lambda \geqslant N^{2} / 2$ will be chosen later.

Following [MN18a, JNPS18], for any integers $r \geqslant 0$ and $\rho \geqslant 1$, we introduce the events $(7)$

$$
\begin{aligned}
\bar{G}_{r}=\bigcap_{j=0}^{r} G_{j}, \quad G_{j}=\left\{v_{t}=u_{t}^{\prime}, \forall t \in(j, j+1]\right\}, \quad F_{r, 0}=\varnothing, \\
F_{r, \rho}=\left\{\sup _{t \in[0, r]}\left(\int_{0}^{t}\left(\left\|u_{s}\right\|_{1}^{2}+\left\|u_{s}^{\prime}\right\|_{1}^{2}\right) \mathrm{d} s-K t\right) \leqslant\|z\|^{2}+\left\|z^{\prime}\right\|^{2}+\rho ;\right. \\
\left.\left\|u_{r}\right\|^{2}+\left\|u_{r}^{\prime}\right\|^{2} \leqslant \rho\right\},
\end{aligned}
$$

where $K$ is the constant in (6.19), and the pairwise disjoint events

$$
A_{0}=G_{0}^{c}, \quad A_{r, \rho}=\left(\bar{G}_{r-1} \cap G_{r}^{c} \cap F_{r, \rho}\right) \backslash F_{r, \rho-1}, r \geqslant 1, \rho \geqslant 1, \quad \tilde{A}=\bar{G}_{+\infty}
$$

We decompose as follows

$$
\begin{aligned}
\mathfrak{P}_{t}^{V} \psi(z)-\mathfrak{P}_{t}^{V} \psi\left(z^{\prime}\right)=\mathbb{E}\left\{\mathbb{I}_{A_{0}}\left[\Xi_{t}^{V} \psi\left(u_{t}\right)-\Xi_{t}^{V} \psi\left(u_{t}^{\prime}\right)\right]\right\} & \\
& +\sum_{r, \rho=1}^{\infty} \mathbb{E}\left\{\mathbb{I}_{A_{r, \rho}}\left[\Xi_{t}^{V} \psi\left(u_{t}\right)-\Xi_{t}^{V} \psi\left(u_{t}^{\prime}\right)\right]\right\} \\
& +\mathbb{E}\left\{\mathbb{I}_{\tilde{A}}\left[\Xi_{t}^{V} \psi\left(u_{t}\right)-\Xi_{t}^{V} \psi\left(u_{t}^{\prime}\right)\right]\right\} \\
= & I_{0}^{t}+\sum_{r, \rho=1}^{\infty} I_{r, \rho}^{t}+\tilde{I}^{t},
\end{aligned}
$$

where

$$
\begin{aligned}
I_{0}^{t} & =\mathbb{E}\left\{\mathbb{I}_{A_{0}}\left[\Xi_{t}^{V} \psi\left(u_{t}\right)-\Xi_{t}^{V} \psi\left(u_{t}^{\prime}\right)\right]\right\}, \\
I_{r, \rho}^{t} & =\mathbb{E}\left\{\mathbb{I}_{A_{r, \rho}}\left[\Xi_{t}^{V} \psi\left(u_{t}\right)-\Xi_{t}^{V} \psi\left(u_{t}^{\prime}\right)\right]\right\}, \\
\tilde{I}^{t} & =\mathbb{E}\left\{\mathbb{I}_{\tilde{A}}\left[\Xi_{t}^{V} \psi\left(u_{t}\right)-\Xi_{t}^{V} \psi\left(u_{t}^{\prime}\right)\right]\right\} .
\end{aligned}
$$

(7) The event $\bar{G}_{r}$ is well defined also for $r=+\infty$. 
In Steps 2 and 3, we estimate $I_{0}^{t}, I_{r, \rho}^{t}$, and $\tilde{I}^{t}$.

Step 2: Estimates for $I_{0}^{t}$ and $I_{r, \rho}^{t}$. - We have following inequalities

$$
\begin{aligned}
\left|I_{0}^{t}\right| & \leqslant C_{1}(R, V)\left\|\mathfrak{P}_{t}^{V} \mathbf{1}\right\|_{R} \mathbb{P}\left(A_{0}\right)^{1 / 2} \\
\left|I_{r, \rho}^{t}\right| & \leqslant C_{2}(R, V) e^{r\|V\|_{\infty}}\left\|\mathfrak{P}_{t}^{V} \mathbf{1}\right\|_{R} \mathbb{P}\left(A_{r, \rho}\right)^{1 / 2}
\end{aligned}
$$

for any integers $r, \rho \geqslant 1$ and $R \geqslant R_{0}$, where $R_{0}$ is the number in Proposition 3.1. Let us prove (4.6), the other inequality is proved in a similar way. First assume that $r+1 \leqslant t$. Using $\psi \leqslant 1$, the positivity of $\Xi_{t}^{V} \psi$, and the Markov property, we derive

$$
\begin{aligned}
I_{r, \rho}^{t} & \leqslant \mathbb{E}\left\{\mathbb{I}_{A_{r, \rho}} \Xi_{t}^{V} \psi\left(u_{t}\right)\right\} \leqslant \mathbb{E}\left\{\mathbb{I}_{A_{r, \rho}} \Xi_{t}^{V}\right\} \\
& =\mathbb{E}\left\{\mathbb{I}_{A_{r, \rho}} \mathbb{E}\left[\Xi_{t}^{V} \mid \mathcal{F}_{r+1}\right]\right\} \leqslant e^{r\|V\|_{\infty}} \mathbb{E}\left\{\mathbb{I}_{A_{r, \rho}}\left(\mathfrak{P}_{t-r-1}^{V} \mathbf{1}\right)\left(u_{r+1}\right)\right\},
\end{aligned}
$$

where $\left\{\mathcal{F}_{t}\right\}$ is the filtration generated by $\left(u_{t}, u_{t}^{\prime}\right)$. Then from the positivity of $V$ and (3.1) it follows that

$$
\mathfrak{P}_{t-r-1}^{V} \mathbf{1}(y) \leqslant \mathfrak{P}_{t}^{V} \mathbf{1}(y) \leqslant M\left\|\mathfrak{P}_{t}^{V} \mathbf{1}\right\|_{R_{0}} \mathfrak{w}(y), \quad y \in H,
$$

so that

$$
\begin{aligned}
I_{r, \rho}^{t} & \leqslant C_{3} e^{r\|V\|_{\infty}}\left\|\mathfrak{P}_{t}^{V} \mathbf{1}\right\|_{R_{0}} \mathbb{E}\left\{\mathbb{I}_{A_{r, \rho}} \mathfrak{w}\left(u_{r}\right)\right\} \\
& \leqslant C_{3} e^{r\|V\|_{\infty}}\left\|\mathfrak{P}_{t}^{V} \mathbf{1}\right\|_{R_{0}}\left\{\mathbb{P}\left(A_{r, \rho}\right) \mathbb{E} \mathfrak{w}^{2}\left(u_{r}\right)\right\}^{1 / 2} .
\end{aligned}
$$

Using this, (6.23), and the symmetry, we obtain (4.6). If $r>t$, then

$$
I_{r, \rho}^{t} \leqslant e^{r\|V\|_{\infty}} \mathbb{P}\left(A_{r, \rho}\right) \leqslant e^{r\|V\|_{\infty}}\left\|\mathfrak{P}_{t}^{V} \mathbf{1}\right\|_{R} \mathbb{P}\left(A_{r, \rho}\right)^{1 / 2},
$$

which implies (4.6) by symmetry.

Step 3: Estimate for $\tilde{I}^{t}$. — Let us show that

$$
\left|\tilde{I}^{t}\right| \leqslant C_{4}(R, V, \lambda, N, \psi)\left\|\mathfrak{P}_{t}^{V} \mathbf{1}\right\|_{R} d .
$$

Indeed, we write

$$
\tilde{I}^{t}=\mathbb{E}\left\{\mathbb{I}_{\tilde{A}} \Xi_{t}^{V}\left[\psi\left(u_{t}\right)-\psi\left(u_{t}^{\prime}\right)\right]\right\}+\mathbb{E}\left\{\mathbb{I}_{\tilde{A}}\left[\Xi_{t}^{V}-\Xi_{t}^{V^{\prime}}\right] \psi\left(u_{t}^{\prime}\right)\right\}=: J_{1}^{t}+J_{2}^{t},
$$

where $\Xi_{t}^{V^{\prime}}:=\exp \left(\int_{0}^{t} V\left(u_{s}^{\prime}\right) \mathrm{d} s\right)$. Then by (6.3), on the event $\tilde{A}$ we have

$$
\left\|\mathrm{P}_{N}\left(u_{s}-u_{s}^{\prime}\right)\right\| \leqslant e^{-\lambda s} d, \quad s \in[0, t] .
$$

Since $\psi \in L_{b}(H)$, we derive from this

$$
\left|J_{1}^{t}\right| \leqslant \mathbb{E}\left\{\mathbb{I}_{\tilde{A}} \Xi_{t}^{V}\left|\psi\left(u_{t}\right)-\psi\left(u_{t}^{\prime}\right)\right|\right\} \leqslant\|\psi\|_{L} e^{-\lambda t}\left\|\mathfrak{P}_{t}^{V} \mathbf{1}\right\|_{R} d \leqslant\|\psi\|_{L}\left\|\mathfrak{P}_{t}^{V} \mathbf{1}\right\|_{R} d .
$$

Similarly, as $V \in L_{b}(H)$,

$$
\begin{aligned}
\left|J_{2}^{t}\right| \leqslant \mathbb{E}\left\{\mathbb{I}_{\tilde{A}}\left|\Xi_{t}^{V}-\Xi_{t}^{V^{\prime}}\right|\right\} & \leqslant \mathbb{E}\left\{\mathbb{I}_{\tilde{A}} \Xi_{t}^{V}\left[\exp \left(\int_{0}^{t}\left|V\left(u_{s}\right)-V\left(u_{s}^{\prime}\right)\right| \mathrm{d} s\right)-1\right]\right\} \\
& \leqslant\left[\exp \left(C_{\lambda, N} \lambda^{-1}\|V\|_{L} d\left(1-e^{-\lambda t}\right)\right)-1\right]\left\|\mathfrak{P}_{t}^{V} \mathbf{1}\right\|_{R} \\
& \leqslant\left[\exp \left(C_{5}(R, V, \lambda, N) d\right)-1\right]\left\|\mathfrak{P}_{t}^{V} \mathbf{1}\right\|_{R} .
\end{aligned}
$$

Recalling that $d \leqslant 1$ and combining the estimates for $J_{1}^{t}$ and $J_{2}^{t}$, we get (4.7). 
Step 4: Uniform equicontinuity of $g_{t}$. - We use the following lemma, which is proved at the end of this subsection.

Lemma 4.3. - For any $\alpha>0$, there is an integer $N_{2}(\alpha) \geqslant 1$ and positive numbers $a$ and $\beta$ such that

$$
\begin{aligned}
\mathbb{P}\left\{A_{0}\right\} & \leqslant C_{6}(R, \lambda, N) d^{a / 2}, \\
\mathbb{P}\left\{A_{r, \rho}\right\} & \leqslant C_{7}(R)\left\{\left(d^{a} e^{-a \alpha r}+\left[\exp \left(C_{8}(R, \lambda, N) d^{a} e^{C^{\prime} \rho-a \alpha r}\right)-1\right]^{1 / 2}\right) \wedge e^{-\beta \rho}\right\}
\end{aligned}
$$

for any $N \geqslant N_{2}(\alpha), \lambda \geqslant N^{2} / 2, R \geqslant 1$, and a universal constant $C^{\prime}>0$.

From (4.4)-(4.9) it follows that, for any $z, z^{\prime} \in X_{R}, t \geqslant 0, R \geqslant R_{0}$, and $\alpha>0$, we have

$$
\begin{aligned}
\left|g_{t}(z)-g_{t}\left(z^{\prime}\right)\right| & \leqslant C_{9}(R, V, \lambda, N, \psi)\left(d^{a / 4}+d\right. \\
& \left.+\sum_{r, \rho=1}^{\infty} e^{r\|V\|_{\infty}}\left\{\left(d^{a / 2} e^{-a \alpha r / 2}+\left[\exp \left(C_{8} d^{a} e^{C^{\prime} \rho-a \alpha r}\right)-1\right]^{1 / 4}\right) \wedge e^{-\beta \rho / 2}\right\}\right),
\end{aligned}
$$

provided that $N \geqslant N_{0} \vee N_{1} \vee N_{2}(\alpha)$ and $\lambda \geqslant N^{2} / 2$. When $d=0$, the series on the right-hand side vanishes. So to prove the uniform equicontinuity of $\left\{g_{t}\right\}$, it suffices to show that the series converges uniformly in $d \in[0,1]$. Since its terms are positive and monotone, it suffices to show the converge for $d=1$ :

$$
\sum_{r, \rho=1}^{\infty} e^{r\|V\|_{\infty}}\left\{\left(e^{-a \alpha r / 2}+\left[\exp \left(C_{8} e^{C^{\prime} \rho-a \alpha r}\right)-1\right]^{1 / 4}\right) \wedge e^{-\beta \rho / 2}\right\}<\infty .
$$

To prove this, we will assume that $\alpha$ is sufficiently large. Let

$$
S_{1}=\left\{(r, \rho) \in \mathbb{N}^{2}: \rho \leqslant \operatorname{a\alpha r} /\left(2 C^{\prime}\right)\right\}, \quad S_{2}=\mathbb{N}^{2} \backslash S_{1} .
$$

Then taking $\alpha>16\|V\|_{\infty} / a$, we see that

$$
\begin{aligned}
& \sum_{(r, \rho) \in S_{1}} e^{r\|V\|_{\infty}}\left(e^{-a \alpha r / 2}+\left[\exp \left(C_{8} e^{C^{\prime} \rho-a \alpha r}\right)-1\right]^{1 / 4}\right) \\
& \leqslant C_{10}(R, N) \sum_{(r, \rho) \in S_{1}} e^{r\|V\|_{\infty}} e^{-a \alpha r / 8} \leqslant C_{11}(R, N) \sum_{r=1}^{\infty} e^{-a \alpha r / 16}<\infty .
\end{aligned}
$$

Choosing $\alpha>8 C^{\prime}\|V\|_{\infty} /(a \beta)$, we get

$$
\sum_{(r, \rho) \in S_{2}} e^{r\|V\|_{\infty}} e^{-\beta \rho / 2} \leqslant C_{12} \sum_{\rho=1}^{\infty} e^{-\beta \rho / 4}<\infty .
$$

These two inequalities show that (4.10) holds.

Proof of Lemma 4.3. - Taking $\varepsilon=d$ in (4.3) and using $d \leqslant 1$, we get

$$
\mathbb{P}\left\{A_{0}\right\} \leqslant d^{a}+2\left[\exp \left(C_{\lambda, N} d^{a} e^{2 C R^{2}}\right)-1\right]^{1 / 2} \leqslant C_{6}(R, \lambda, N) d^{a / 2}
$$


for $N \geqslant N_{1}$ and $\lambda \geqslant N^{2} / 2$. This gives (4.8). From the inclusion $A_{r, \rho} \subset F_{r, \rho-1}^{c}$ and inequalities (6.19) and (6.21) it follows that

$$
\mathbb{P}\left\{A_{r, \rho}\right\} \leqslant C_{13}(R) e^{-\beta \rho},
$$

where $\beta:=\gamma_{0} / 2$. By Proposition 6.1 , on the event $A_{r, \rho}$ we have

$$
\left\|u_{r}-u_{r}^{\prime}\right\| \leqslant \exp \left(-\alpha r+c\left(\|z\|^{2}+\left\|z^{\prime}\right\|^{2}+\rho\right)\right) d \leqslant C_{13}(R) e^{-\alpha r+c \rho} d,
$$

provided that $N \geqslant N_{1}^{\prime}(\alpha):=\sqrt{\alpha+c K}$ and $\lambda \geqslant N^{2} / 2$. Recall that on the same event we have also

$$
\left\|u_{r}\right\|^{2}+\left\|u_{r}^{\prime}\right\|^{2} \leqslant \rho .
$$

Using the Markov property, (4.3) with $\varepsilon=d e^{-\alpha r}$, (4.12) and (4.13), we obtain

$$
\begin{aligned}
& \mathbb{P}\left\{A_{r, \rho}\right\} \leqslant \mathbb{P}\left\{\bar{G}_{r-1} \cap G_{r}^{c} \cap F_{r, \rho}\right\}=\mathbb{E}\left\{\mathbb{I}_{\bar{G}_{r-1} \cap F_{r, \rho}} \mathbb{E}\left(\mathbb{I}_{G_{r}^{c}} \mid \mathcal{F}_{r}\right)\right\} \\
& \leqslant d^{a} e^{-a \alpha r}+2 \mathbb{E}\left\{\mathbb{I}_{\bar{G}_{r-1} \cap F_{r, \rho}}\left[\exp \left(C_{\lambda, N} d^{a-2} e^{-(a-2) \alpha r}\left\|u_{r}-u_{r}^{\prime}\right\|^{2} e^{C\left(\left\|u_{r}\right\|+\left\|u_{r}^{\prime}\right\|\right)}\right)-1\right]^{1 / 2}\right\} \\
& \leqslant d^{a} e^{-a \alpha r}+2\left[\exp \left(C_{8}(R, \lambda, N) d^{a} e^{C^{\prime} \rho-a \alpha r}\right)-1\right]^{1 / 2} .
\end{aligned}
$$

Combining this with (4.11) and taking $N \geqslant N_{2}(\alpha):=N_{1} \vee N_{1}^{\prime}(\alpha)$ and $\lambda \geqslant N^{2} / 2$, we get the required inequality (4.9).

\section{Proof of Theorem 2.1}

The results of Sections 3 and 4 show that the conditions of Theorem 6.6 are satisfied if we choose

$$
\begin{gathered}
P_{t}^{V}(u, \Gamma)=\left(\mathfrak{P}_{t}^{V *} \delta_{u}\right)(\Gamma), \quad X=H, \quad X_{R}=B_{H^{1}}(R), \quad R \geqslant R_{0}, \\
\mathfrak{w}(u)=\mathfrak{w}_{m}(u)=1+\|u\|^{2 m}, \mathcal{C}=\mathcal{V}, \quad V \in \mathcal{V}
\end{gathered}
$$

with sufficiently large $m$ and $R_{0}$. Thus there are eigenvectors $\mu_{V} \in \mathcal{P}(H)$ and $h_{V} \in L_{\mathfrak{w}}^{\infty}(H)$ corresponding to an eigenvalue $\lambda_{V}>0$. Moreover, for any $R \geqslant 1$, the restriction of $h_{V}$ to $X_{R}$ is continuous and strictly positive, so $h_{V}: H^{1} \rightarrow \mathbb{R}$ is continuous and strictly positive. As $\mathbb{P}_{u}\left\{u_{1} \in H^{1}\right\}=1$ and $h(u)=\lambda_{V}^{-1} \mathfrak{P}_{1}^{V} h_{V}(u)$, we have

$$
h_{V}(u) \geqslant \lambda_{V}^{-1} e^{-\|V\|_{\infty}} \mathbb{E}_{u} h_{V}\left(u_{1}\right)>0 \quad u \in H .
$$

The continuity of $h_{V}: H \rightarrow \mathbb{R}$ follows from the uniform convergence in (2.4), and the uniqueness of $\mu_{V}$ and $h_{V}$ from (2.4) and (2.5). The proof of (2.4) is carried out in Steps 1-3, and that of (2.6) in Step 4. Convergence (2.5) follows immediately from (2.4).

Step 1: Proof of (2.4) for $f \in \mathcal{V}$. - In view of (6.32), for any $f \in \mathcal{V}$, we have limit (2.4) in $C\left(X_{R}\right) \cap L^{1}\left(H, \mu_{V}\right)$. We claim that this limit holds also in $C\left(B_{H}(R)\right)$ for any $R \geqslant 1$. Indeed, it suffices to check condition (6.33) with $B=B_{H}(R)$ and $s=1$, i.e.,

$$
A_{R, r}:=\sup _{u \in B_{H}(R)} \int_{H \backslash X_{r}} \mathfrak{w}_{m}(v) P_{1}^{V}(u, \mathrm{~d} v) \rightarrow 0 \quad \text { as } r \rightarrow \infty .
$$


From the Poincaré inequality and (6.24) it follows that

$$
\begin{aligned}
A_{R, r} & \leqslant r^{-2} \sup _{u \in B_{H}(R)} \mathbb{E}_{u}\left\{\mathfrak{w}_{m}\left(u_{1}\right)\left\|u_{1}\right\|_{1}^{2} \Xi_{1}^{V}\right\} \\
& \leqslant r^{-2} e^{\|V\|_{\infty}} \sup _{u \in B_{H}(R)} \mathbb{E}_{u}\left\{\left(1+\alpha_{1}^{-m}\left\|u_{1}\right\|_{1}^{2 m}\right)\left\|u_{1}\right\|_{1}^{2}\right\} \\
& \leqslant r^{-2} C(m) R^{8(m+1)} \rightarrow 0 \quad \text { as } r \rightarrow \infty .
\end{aligned}
$$

This implies (2.4) for $f \in \mathcal{V}$.

Step 2: Proof of (2.4) for $f \in C_{b}(H)$. - For any $n \geqslant 1$, let $\tilde{f}_{n} \in L_{b}(H)$ be such that

$$
\sup _{u \in B_{H}(n)}\left|\tilde{f}_{n}(u)-f(u)\right| \leqslant \frac{1}{n} .
$$

Then the functions $f_{n}=\tilde{f}_{n} \circ \mathrm{P}_{n}$ belong to the space $\mathcal{V}$, satisfy $\left\|f_{n}\right\|_{\infty} \leqslant\|f\|_{\infty}$ and $f_{n} \rightarrow f$ as $n \rightarrow \infty$, uniformly on compact subsets of $H$. Setting

$$
\Delta_{t}(g)=\sup _{u \in B_{H}(R)}\left|\lambda_{V}^{-t} \mathfrak{P}_{t}^{V} g(u)-\left\langle g, \mu_{V}\right\rangle h_{V}(u)\right|, \quad\|g\|_{0, R}=\sup _{u \in B_{H}(R)}|g(u)|,
$$

for any $t \geqslant 0$ and $n \geqslant 1$, we write

$$
\Delta_{t}(f) \leqslant \Delta_{t}\left(f_{n}\right)+\left\|h_{V}\right\|_{0, R}\left|\left\langle f-f_{n}, \mu_{V}\right\rangle\right|+\lambda_{V}^{-t}\left\|\mathfrak{P}_{t}^{V}\left(f-f_{n}\right)\right\|_{0, R} .
$$

Since $f_{n} \in \mathcal{V}$, the first term on the right-hand side of this inequality goes to zero as $k \rightarrow \infty$ for any fixed $n \geqslant 1$. The Lebesgue theorem on dominated convergence implies that $\left|\left\langle f-f_{n}, \mu_{V}\right\rangle\right| \rightarrow 0$ as $n \rightarrow \infty$. Thus, the required convergence will be established if we show that

$$
\sup _{t \geqslant 1} \lambda_{V}^{-t}\left\|\mathfrak{P}_{t}^{V}\left(f-f_{n}\right)\right\|_{0, R} \rightarrow 0 \quad \text { as } n \rightarrow \infty .
$$

To prove this limit, we take any $\rho>0$ and write

$$
\left\|\mathfrak{P}_{t}^{V}\left(f-f_{n}\right)\right\|_{0, R} \leqslant J_{1}(t, n, \rho)+J_{2}(t, n, \rho),
$$

where

$$
J_{1}(t, n, \rho)=\left\|\mathfrak{P}_{t}^{V}\left(\left(f-f_{n}\right) \mathbb{I}_{X_{\rho}}\right)\right\|_{0, R}, \quad J_{2}(t, n, \rho)=\left\|\mathfrak{P}_{t}^{V}\left(\left(f-f_{n}\right) \mathbb{I}_{X_{\rho}^{c}}\right)\right\|_{0, R} .
$$

By (3.2), we have

$$
J_{1}(t, n, \rho) \leqslant \varepsilon(n, \rho)\left\|\mathfrak{P}_{t}^{V} \mathbf{1}\right\|_{0, R} \leqslant \varepsilon(n, \rho) C_{R}\left\|\mathfrak{P}_{t}^{V} \mathbf{1}\right\|_{R_{0}},
$$

where $\varepsilon(n, \rho)=\left\|f-f_{n}\right\|_{X_{\rho}} \rightarrow 0$ as $n \rightarrow \infty$. Convergence (2.4) with $f=1$ implies that

$$
\text { the set }\left\{\lambda_{V}^{-t}\left\|\mathfrak{P}_{t}^{V} \mathbf{1}\right\|_{R_{0}}\right\}_{t \geqslant 0} \text { is bounded in } \mathbb{R} \text {. }
$$

It follows that

$$
\sup _{t \geqslant 0} \lambda_{V}^{-t} J_{1}(t, n, \rho) \rightarrow 0 \quad \text { as } n \rightarrow \infty .
$$

To estimate $J_{2}$, we use (3.8). For any $\rho, n \geqslant 1$ and $t \geqslant 0$, we have

$$
\begin{aligned}
\lambda_{V}^{-t} J_{2}(t, n, \rho) & \leqslant 2\|f\|_{\infty} \rho^{-2} \lambda_{V}^{-t}\left\|\mathfrak{P}_{t}^{V} F\right\|_{0, R} \\
& \leqslant C_{R}\|f\|_{\infty} \rho^{-2} \lambda_{V}^{-t}\left\|\mathfrak{P}_{t}^{V} \mathbf{1}\right\|_{R_{0}} .
\end{aligned}
$$


By (5.3), the right-hand side of this inequality goes to zero as $\rho \rightarrow \infty$, uniformly with respect to $t \geqslant 1$. Combining this with (5.4), we see that supremum over $t \geqslant 1$ of the right-hand side of (5.2) can be made arbitrarily small by choosing first $\rho>0$ and then $n \geqslant 1$ sufficiently large. This proves (5.1).

Step 3: Proof of (2.4) for $f \in C_{\mathfrak{m}}(H)$. - We use again an approximation argument. Let us fix any $\varkappa \in\left(0, \gamma_{0}\right)$ and $f \in C_{\mathfrak{m}}(H)$ with $\mathfrak{m}=\mathfrak{m}_{\varkappa}$. We define a sequence $\left\{f_{n}\right\}$ by the relation $f_{n}=f^{+} \wedge n-f^{-} \wedge n$. Then $f_{n} \in C_{b}(H),\left|f_{n}\right| \leqslant|f|$ for any $n \geqslant 1$, and $f_{n} \rightarrow f$ in $L_{\mathfrak{m}^{\prime}}^{\infty}(H)$ with $\mathfrak{m}^{\prime}=\mathfrak{m}_{\varkappa^{\prime}}$ for any $\varkappa^{\prime} \in\left(\varkappa, \gamma_{0}\right)$. Furthermore, in view of (2.4) and the Lebesgue theorem on dominated convergence, we have

$$
\begin{gathered}
\sup _{u \in B_{H}(R)}\left|\lambda_{V}^{-t} \mathfrak{P}_{t}^{V} f_{n}(u)-\left\langle f_{n}, \mu_{V}\right\rangle h_{V}(u)\right| \rightarrow 0 \quad \text { as } t \rightarrow \infty \text { for any fixed } n \geqslant 1, \\
\left|\left\langle f-f_{n}, \mu_{V}\right\rangle\right| \rightarrow 0 \text { as } n \rightarrow \infty .
\end{gathered}
$$

Thus, as in the previous step, it suffices to prove that

$$
\sup _{t \geqslant 0} \lambda_{V}^{-t}\left\|\mathfrak{P}_{t}^{V}\left(f-f_{n}\right)\right\|_{0, R} \rightarrow 0 \quad \text { as } n \rightarrow \infty
$$

To see this, we use (3.7) for $\mathfrak{m}^{\prime}$ :

$$
\left\|\mathfrak{P}_{t}^{V}\left(f-f_{n}\right)\right\|_{0, R} \leqslant \varepsilon_{n}\left\|\mathfrak{P}_{t}^{V} \mathfrak{m}^{\prime}\right\|_{0, R} \leqslant C_{R} \varepsilon_{n}\left\|\mathfrak{P}_{t}^{V} \mathbf{1}\right\|_{R_{0}}
$$

where $\varepsilon_{n}=\left\|f-f_{n}\right\|_{L_{\mathfrak{w}^{\prime}}^{\infty}} \rightarrow 0$ as $n \rightarrow \infty$. Combining this with (5.3), we get (5.5).

Step 4: Proof of (2.6). - In view of (2.4), it suffices to show that

$$
\sup _{(t, \nu) \in \mathbb{R}_{+} \times \Lambda\left(\varkappa^{\prime}, M\right)}\left\{\int_{B_{H}(R)^{c}}\left|\lambda_{V}^{-t} \mathfrak{P}_{t}^{V} f-\left\langle f, \mu_{V}\right\rangle h_{V}\right| \nu(\mathrm{d} u)\right\} \rightarrow 0 \text { as } R \rightarrow \infty .
$$

By (3.7) and (5.3), we have

$$
\left\|\mathfrak{P}_{t}^{V} f\right\|_{L_{\mathfrak{m}}^{\infty}} \leqslant C_{1}\left\|\mathfrak{P}_{t}^{V} \mathbf{1}\right\|_{R_{0}} \leqslant C_{2} \lambda_{V}^{t} \text { for all } t \geqslant 0 .
$$

It follows that

$$
\left|\lambda_{V}^{-k} \mathfrak{P}_{t}^{V} f(u)\right| \leqslant C_{3} \mathfrak{m}_{\varkappa}(u) .
$$

Since $\varkappa<\varkappa^{\prime}, h_{V} \in C_{\mathfrak{w}}(H)$, and

$$
\sup _{\nu \in \Lambda\left(\varkappa^{\prime}, M\right)} \int_{B_{H}(R)^{c}} \mathfrak{m}_{\varkappa}(u) \nu(\mathrm{d} u) \leqslant M e^{\left(\varkappa-\varkappa^{\prime}\right) R^{2}} \rightarrow 0 \quad \text { as } R \rightarrow \infty,
$$

we obtain (5.6). This completes the proof of Theorem 2.1.

\section{Appendix}

\subsection{The Foiaş-Prodi estimate}

Let us take any numbers $T, \lambda>0$, any function $\varphi \in L^{2}([0, T] ; H)$, any integer $N \geqslant 1$, and consider the equations

$$
\begin{aligned}
\dot{u}+B(u)+L u & =h(x)+\partial_{t} \varphi(t, x), \\
\dot{v}+B(v)+L v+\mathrm{P}_{N}[\lambda(v-u)+B(u)-B(v)] & =h(x)+\partial_{t} \varphi(t, x),
\end{aligned}
$$


where $\mathrm{P}_{N}$ is the orthogonal projection in $H$ onto the space $H_{N}$ defined by (1.11). The following result is a version of the Foiaş-Prodi estimate obtained in [FP67]; see also [KS12, Section 2.1.8] for a similar result for the Navier-Stokes system (with different equation instead of (6.2)) and [MN18a, Section 7.3] for the damped nonlinear wave equation.

Proposition 6.1. - Let $u, v \in C([0, T] ; H) \cap L^{2}\left([0, T] ; H^{1}\right)$ be solutions of $(6.1)$ and (6.2) issued from $z$ and $z^{\prime}$, respectively. Then

$$
\left\|\mathrm{P}_{N}\left(u_{t}-v_{t}\right)\right\| \leqslant e^{-\lambda t}\left\|\mathbf{P}_{N}\left(z-z^{\prime}\right)\right\|, \quad t \in[0, T] .
$$

If we assume additionally that

$$
\int_{0}^{t}\left(\left\|u_{s}\right\|_{1}^{2}+\left\|v_{s}\right\|_{1}^{2}\right) \mathrm{d} s \leqslant \rho+K t, \quad t \in[0, T]
$$

for some numbers $\rho>0$ and $K>0$, then for any $\alpha>0$, we have

$$
\left\|u_{t}-v_{t}\right\| \leqslant C_{\lambda, N} e^{-\alpha t+c \rho}\left\|z-z^{\prime}\right\|, \quad t \in[0, T],
$$

provided that $2 \lambda>N^{2} \geqslant \alpha+c K$. Here $c>0$ is an absolute constant and $C_{\lambda, N}$ is a constant depending on $\lambda$ and $N$.

Proof.

Step 1: Proof of (6.3). - Let us set $y=\mathrm{P}_{N}(u-v)$. Then

$$
\dot{y}+L y+\lambda y=0 .
$$

Taking the scalar product in $H$ of this equation with $y$, we obtain

$$
\frac{1}{2} \frac{\mathrm{d}}{\mathrm{d} t}\|y\|^{2}+\|y\|_{1}^{2}+\lambda\|y\|^{2}=0 .
$$

Hence

which implies (6.3).

$$
\frac{\mathrm{d}}{\mathrm{d} t}\|y\|^{2}+2 \lambda\|y\|^{2} \leqslant 0
$$

Step 2: Proof of (6.5). - Let $w=u-v$. Then

$$
\dot{w}+L w+\lambda \mathrm{P}_{N} w+\mathrm{Q}_{N}[B(u)-B(v)]=0,
$$

where $\mathrm{Q}_{N}=1-\mathrm{P}_{N}$. For any $a, b \in H^{1}$, let us set $B(a, b)=\Pi(\langle a, \nabla\rangle b)$. Taking the scalar product of (6.6) with $w$, and using the equality

$$
B(v)-B(u)=B(v, w)+B(w, u),
$$

we get

$$
\begin{aligned}
\frac{1}{2} \frac{\mathrm{d}}{\mathrm{d} t}\|w\|^{2}+\|w\|_{1}^{2}+\lambda\left\|\mathrm{P}_{N} w\right\|^{2} & =\left\langle B(v)-B(u), \mathrm{Q}_{N} w\right\rangle \\
& =\left\langle B(v, w), \mathrm{Q}_{N} w\right\rangle+\left\langle B(w, u), \mathrm{Q}_{N} w\right\rangle \\
& =: I_{1}+I_{2} .
\end{aligned}
$$

Using the identity

$$
\langle B(a, b), b\rangle=0, \quad a, b \in H^{1}
$$


and the Hölder inequality, we obtain

$$
\begin{aligned}
\left|I_{1}\right|=\mid\left\langle B\left(v, \mathrm{P}_{N} w\right),\right. & \left.\mathrm{Q}_{N} w\right\rangle\left|\leqslant C_{1} \int_{\mathbb{T}^{2}}\right| v\left\|\nabla \mathrm{P}_{N} w\right\| \mathrm{Q}_{N} w \mid \mathrm{d} x \\
& \leqslant C_{1}\|v\|\left\|\nabla \mathrm{P}_{N} w\right\|_{\infty}\|w\| \leqslant \frac{1}{2}\left\|\nabla \mathrm{P}_{N} w\right\|_{\infty}^{2}+C_{2}\|v\|^{2}\|w\|^{2} .
\end{aligned}
$$

To estimate $I_{2}$, we use the Hölder inequality, the inclusion $H^{\frac{1}{2}} \subset L^{4}$, and the interpolation inequality $\|a\|_{1 / 2}^{2} \leqslant\|a\|\|a\|_{1}$ :

$$
\begin{aligned}
& \left|I_{2}\right|=\left|\left\langle B(w, u), \mathrm{Q}_{N} w\right\rangle\right| \leqslant C_{3} \int_{\mathbb{T}^{2}}|w|\left|\nabla u \| \mathrm{Q}_{N} w\right| \mathrm{d} x \\
& \quad \leqslant C_{3}\|w\|_{L^{4}}\|u\|_{1}\left\|\mathrm{Q}_{N} w\right\|_{L^{4}} \leqslant C_{4}\|w\|\|w\|_{1}\|u\|_{1} \leqslant \frac{1}{2}\|w\|_{1}^{2}+C_{5}\|w\|^{2}\|u\|_{1}^{2} .
\end{aligned}
$$

Combining this with (6.8) and (6.9), and using the Poincaré inequality

$$
N\left\|\mathrm{Q}_{N} w\right\| \leqslant\|w\|_{1},
$$

we get

$$
\frac{\mathrm{d}}{\mathrm{d} t}\|w\|^{2}+\left(l-c_{1}\left(\|u\|_{1}^{2}+\|v\|_{1}^{2}\right)\right)\|w\|^{2} \leqslant\left\|\nabla \mathrm{P}_{N} w\right\|_{\infty}^{2},
$$

where $l=\min \left\{N^{2}, 2 \lambda\right\}$. From (6.3) we deduce that

$$
\left\|\nabla \mathrm{P}_{N} w_{t}\right\|_{\infty}^{2} \leqslant C_{N} e^{-2 \lambda t}\left\|w_{0}\right\|^{2}
$$

Hence, (6.10) and (6.4) imply that

$$
\left\|w_{t}\right\|^{2} \leqslant\left(1+C_{N} \int_{0}^{t} e^{(l-2 \lambda) s} \mathrm{~d} s\right)\left\|w_{0}\right\|^{2} \exp \left(-l t+c_{1}(\rho+K t)\right) .
$$

Choosing $\lambda$ and $N$ such that $2 \lambda>N^{2} \geqslant 2 \alpha+c_{1} K$, we get (6.5) with $c=c_{1} / 2$.

\subsection{Proof of Proposition 4.2}

We closely follow the arguments of the proof of a similar result from Section 7.3 of [MN18a] in the case of the nonlinear wave equation (see also [KS12, Section 3.3.3]).

Note that inequality (4.2) concerns the laws of the solutions and not the solutions themselves. Thus we can choose the underlying probability space $(\Omega, \mathcal{F}, \mathbb{P})$. We assume that $\Omega$ is the space $C\left(\mathbb{R}_{+} ; \mathbb{R}\right)$ endowed with the topology of uniform convergence on bounded intervals, $\mathbb{P}$ is the law of the Wiener process $W$ in (1.3), and $\mathcal{F}$ is the completion of the Borel $\sigma$-algebra of $\Omega$ with respect to $\mathbb{P}$. We define a stopping time by

$$
\tau^{u}=\inf \left\{t \geqslant 0: \mathcal{E}^{u}(t) \geqslant\|z\|^{2}+K t+\rho\right\},
$$

where $\mathcal{E}^{u}(t)$ is the functional and $K$ is the number in Lemma 6.3 , and $\rho>0$ is a constant to be chosen later. The stopping times $\tau^{u^{\prime}}$ and $\tau^{v}$ are defined in a similar way. Then by inequality (6.19), we have

$$
\mathbb{P}\left\{\tau^{u}<\infty\right\}+\mathbb{P}\left\{\tau^{u^{\prime}}<\infty\right\} \leqslant 2 e^{-\gamma_{0} \rho} .
$$


We define a transformation $\Lambda: \Omega \rightarrow \Omega$ by

$$
\Lambda(\omega)(t)=\omega(t)-\int_{0}^{t} \varphi(s, \omega) \mathrm{d} s, \quad \varphi(t, \omega)=\mathbb{I}_{\{t \leqslant \tilde{\tau}\}} \mathrm{P}_{N}[\lambda(v-u)+B(u)-B(v)],
$$

where $\tilde{\tau}=\tau^{u} \wedge \tau^{u^{\prime}} \wedge \tau^{v}$ and $\mathbb{I}_{\{t \leqslant \tilde{\tau}\}}$ is the indicator function of the interval $[0, \tilde{\tau}]$. We use the following result, whose proof is given at the end of this section.

Lemma 6.2. - There is an integer $N_{1} \geqslant 1$ such that for any numbers $N \geqslant N_{1}$, $\lambda \geqslant N^{2} / 2$, and $\rho>0$ and any initial points $z, z^{\prime} \in H$, we have

$$
\left\|\Lambda_{*} \mathbb{P}-\mathbb{P}\right\|_{\text {var }} \leqslant\left[\exp \left(C_{\lambda, N}\left\|z-z^{\prime}\right\|^{2} e^{C\left(\|z\|^{2}+\left\|z^{\prime}\right\|^{2}+\rho\right)}\right)-1\right]^{1 / 2},
$$

where $\Lambda_{*} \mathbb{P}$ stands for the image of $\mathbb{P}$ under $\Lambda$, and $C$ and $C_{\lambda, N}$ are positive constants not depending on $\rho, z, z^{\prime}$.

Let us introduce auxiliary processes $y_{u^{\prime}}$ and $y_{v}$ in $H$ defined as follows: for $t \leqslant \tilde{\tau}$ they coincide with the processes $u^{\prime}$ and $v$, respectively, while for $t \geqslant \tilde{\tau}$ and $\tilde{\tau}<\infty$ they are zero. With probability 1, we have

$$
y_{v}(t, \omega)=y_{u^{\prime}}(t, \Lambda(\omega)), \quad t \in J .
$$

Let us denote by $u_{1}^{\prime}$ and $v_{1}$ the restrictions of $u^{\prime}(t)$ and $v(t)$ to $J$. Then

$$
\begin{aligned}
& \left\|\nu\left(z, z^{\prime}\right)-\nu^{\prime}\left(z^{\prime}\right)\right\|_{v a r}=\sup _{\Gamma}\left|\mathbb{P}\left\{v_{1} \in \Gamma\right\}-\mathbb{P}\left\{u_{1}^{\prime} \in \Gamma\right\}\right| \\
& \quad \leqslant \mathbb{P}\{\tilde{\tau}<\infty\}+\sup _{\Gamma}\left|\mathbb{P}\left\{v_{1} \in \Gamma, \tilde{\tau}=\infty\right\}-\mathbb{P}\left\{u_{1}^{\prime} \in \Gamma, \tilde{\tau}=\infty\right\}\right|=\mathcal{L}_{1}+\mathcal{L}_{2},
\end{aligned}
$$

where the supremum is taken over all Borel subsets of $C(J ; H)$. Note that

$$
\mathcal{L}_{2} \leqslant\left\|\Lambda_{*} \mathbb{P}-\mathbb{P}\right\|_{\text {var }}
$$

Further, we have

$$
\mathcal{L}_{1} \leqslant \mathbb{P}\left\{\tau^{v}<\infty, \tau^{u} \wedge \tau^{u^{\prime}}=\infty\right\}+\mathbb{P}\left\{\tau^{u}<\infty\right\}+\mathbb{P}\left\{\tau^{u^{\prime}}<\infty\right\} .
$$

Moreover, thanks to (6.13),

$$
\begin{aligned}
\mathbb{P}\left\{\tau^{v}<\infty, \tau^{u} \wedge \tau^{u^{\prime}}=\infty\right\} & \leqslant \mathbb{P}\left\{\tau^{y_{v}}<\infty\right\}=\Lambda_{*} \mathbb{P}\left\{\tau^{y_{u^{\prime}}}<\infty\right\} \\
& \leqslant \mathbb{P}\left\{\tau^{y_{u^{\prime}}}<\infty\right\}+\left\|\Lambda_{*} \mathbb{P}-\mathbb{P}\right\|_{\text {var }} \\
& \leqslant \mathbb{P}\left\{\tau^{u^{\prime}}<\infty\right\}+\left\|\Lambda_{*} \mathbb{P}-\mathbb{P}\right\|_{\text {var }} .
\end{aligned}
$$

Combining last four inequalities, we infer that

$$
\left\|\nu\left(z, z^{\prime}\right)-\nu^{\prime}\left(z^{\prime}\right)\right\|_{\text {var }} \leqslant 2\left(\mathbb{P}\left\{\tau^{u}<\infty\right\}+\mathbb{P}\left\{\tau^{u^{\prime}}<\infty\right\}+\left\|\Lambda_{*} \mathbb{P}-\mathbb{P}\right\|_{\text {var }}\right) .
$$

Finally using this with inequalities (6.11) and (6.12), we get

$$
\left\|\nu\left(z, z^{\prime}\right)-\nu^{\prime}\left(z^{\prime}\right)\right\|_{v a r} \leqslant 4 e^{-\gamma_{0} \rho}+2\left[\exp \left(C_{\lambda, N}\left\|z-z^{\prime}\right\|^{2} e^{C\left(\|z\|^{2}+\left\|z^{\prime}\right\|^{2}+\rho\right)}\right)-1\right]^{1 / 2} .
$$

Choosing $a=2 \gamma_{0} /\left(\gamma_{0}+1\right)$ and $\rho=-\gamma_{0}^{-1} a \ln \left(\varepsilon / 4^{1 / a}\right)$, we obtain (4.2). 


\section{Proof of Lemma 6.2 .}

Step 1: Girsanov theorem. - We write $\Omega=\Omega_{N} \dot{+} \Omega_{N}^{\perp}$, where $\Omega_{N}=C\left(\mathbb{R}_{+} ; H_{N}\right)$ and $\Omega_{N}^{\perp}=C\left(\mathbb{R}_{+} ; H_{N}^{\perp}\right)$. For any $\omega=\omega_{1} \dot{+} \omega_{2} \in \Omega$, we write $\omega=\left(\omega_{1}, \omega_{2}\right) \in \Omega_{N} \times \Omega_{N}^{\perp}$. Then the transformation $\Lambda$ can be written as $\Lambda(\omega)=\left(\Upsilon(\omega), \omega_{2}\right)$, where $\Upsilon: \Omega \rightarrow \Omega_{N}$ is given by

$$
\Upsilon(\omega)(t)=\omega_{1}(t)+\int_{0}^{t} \varphi(s, \omega) \mathrm{d} s .
$$

It is not difficult to see that

$$
\left\|\Lambda_{*} \mathbb{P}-\mathbb{P}\right\|_{v a r} \leqslant \int_{\Omega_{N}^{\perp}}\left\|\Upsilon_{*}\left(\mathbb{P}_{N}, \omega_{2}\right)-\mathbb{P}_{N}\right\|_{v a r} \mathbb{P}_{N}^{\perp}\left(\mathrm{d} \omega_{2}\right),
$$

where $\mathbb{P}_{N}$ and $\mathbb{P}_{N}^{\perp}$ are the images of $\mathbb{P}$ under the projections $\hat{\mathrm{P}}_{N}: \Omega \rightarrow \Omega_{N}$ and $\hat{\mathrm{Q}}_{N}: \Omega \rightarrow \Omega_{N}^{\perp}$, respectively. Let

$$
X=\omega_{1}(t), \quad \hat{X}=\omega_{1}(t)+\int_{0}^{t} \varphi(s, \omega) \mathrm{d} s .
$$

Then $\mathbb{P}_{N}$ coincides with the law $\mathcal{D}(X)$ of the random variable $X$ and $\Upsilon_{*}\left(\mathbb{P}_{N}, \omega_{2}\right)$ coincides with that of $\hat{X}$. By the Girsanov theorem (see [KS12, Theorem A.10.1]), we have

$$
\|\mathcal{D}(\hat{X})-\mathcal{D}(X)\|_{\text {var }} \leqslant \frac{1}{2}\left(\left(\mathbb{E} \exp \left[6 \max _{1 \leqslant j \leqslant N} b_{j}^{-1} \int_{0}^{\infty}\|\varphi(t)\|^{2} \mathrm{~d} t\right]\right)^{\frac{1}{2}}-1\right)^{\frac{1}{2}}
$$

provided that the Novikov condition

$$
\mathbb{E} \exp \left(p \int_{0}^{\infty}\|\varphi(t)\|^{2} \mathrm{~d} t\right)<\infty \quad \text { for any } p>0
$$

is satisfied. In Step 2, we show that

$$
\mathbb{E} \exp \left(p \int_{0}^{\infty}\|\varphi(t)\|^{2} \mathrm{~d} t\right) \leqslant \exp \left(C_{p, \lambda, N}\left\|z-z^{\prime}\right\|^{2} e^{C\left(\|z\|^{2}+\left\|z^{\prime}\right\|^{2}+\rho\right)}\right)
$$

for any $p>0$. Clearly, this and (6.14) imply (6.12).

Step 2: Proof of (6.15). - By Proposition 6.1, the following inequalities hold

$$
\begin{aligned}
\left\|\mathrm{P}_{N}\left(u_{t}-v_{t}\right)\right\| & \leqslant e^{-\lambda t}\left\|\mathrm{P}_{N}\left(z-z^{\prime}\right)\right\|, \quad t \geqslant 0, \\
\left\|u_{t}-v_{t}\right\| & \leqslant C_{1} e^{-t+c\left(\|z\|^{2}+\left\|z^{\prime}\right\|^{2}+\rho\right)}\left\|z-z^{\prime}\right\|, \quad t \in[0, \tilde{\tau}],
\end{aligned}
$$

if $2 \lambda>N^{2} \geqslant 1+c K$. We have

$$
\begin{aligned}
& \mathbb{E} \exp \left(p \int_{0}^{\infty}\|\varphi(t)\|^{2} \mathrm{~d} t\right)=\mathbb{E} \exp \left(p \int_{0}^{\tilde{\tau}}\|\varphi(t)\|^{2} \mathrm{~d} t\right) \\
& \leqslant \mathbb{E} \exp \left(C_{2} \int_{0}^{\tilde{\tau}}\left(\left\|\mathrm{P}_{N}[u-v]\right\|^{2}+\left\|\mathrm{P}_{N}[B(u)-B(v)]\right\|^{2}\right) \mathrm{d} t\right) .
\end{aligned}
$$

Integrating by parts and using the Hölder inequality, we see that

$$
\left|\left\langle B(a, b), e_{j}\right\rangle\right| \leqslant C_{j}^{\prime}\|a\|_{1}\|b\|, \quad a, b \in H^{1}, j \geqslant 1 .
$$


Combining this with (6.7) and (6.16)-(6.18), we get

$$
\begin{aligned}
& \mathbb{E} \exp \left(p \int_{0}^{\infty}\|\varphi(t)\|^{2} \mathrm{~d} t\right) \\
& \quad \leqslant \mathbb{E} \exp \left(C_{3}\left\|z-z^{\prime}\right\|^{2} \int_{0}^{\infty} e^{-t+c\left(\|z\|^{2}+\left\|z^{\prime}\right\|^{2}+\rho\right)}\left(1+\|z\|^{2}+\left\|z^{\prime}\right\|^{2}+K t+\rho\right)^{2} \mathrm{~d} t\right) \\
& \quad \leqslant \mathbb{E} \exp \left(C_{4}\left\|z-z^{\prime}\right\|^{2} \int_{0}^{\infty} e^{-t / 2+2 c\left(\|z\|^{2}+\left\|z^{\prime}\right\|^{2}+\rho\right)} \mathrm{d} t\right) \\
& \quad=\exp \left(2 C_{4}\left\|z-z^{\prime}\right\|^{2} e^{C\left(\|z\|^{2}+\left\|z^{\prime}\right\|^{2}+\rho\right)}\right) .
\end{aligned}
$$

This proves (6.15).

\subsection{A priori estimates}

The following lemma gathers some standard a priori estimates for the solutions of the stochastic Navier-Stokes system. The reader is referred to Section 2.4.2 in [KS12] for more general results.

Lemma 6.3. - Assume ${ }^{(8)}$ that $\mathfrak{B}_{1}<\infty, h \in H^{1}$, and $u_{t}$ is a solution of (1.5) issued from $u \in H$. Then we have the following estimates.

Exponential moments. - There are numbers $\gamma_{0}=\gamma_{0}\left(\mathfrak{B}_{0}\right)>0$ and $K=$ $K\left(\mathfrak{B}_{0},\|h\|\right)>0$ such that for any $\varkappa \in\left(0, \gamma_{0}\right)$,

$$
\begin{aligned}
& \mathbb{P}_{u}\left\{\sup _{t \geqslant 0}(\mathcal{E}(t)-K t) \geqslant\|u\|^{2}+\rho\right\} \leqslant e^{-\gamma_{0} \rho}, \quad \rho \geqslant 0, \\
& \mathbb{E}_{u} e^{\varkappa \mathcal{E}(t)} \leqslant C_{1}\left(\varkappa, \mathfrak{B}_{0}\right) e^{\varkappa\left(K t+\|u\|^{2}\right)}, \\
& \mathbb{E}_{u} \exp \left(\varkappa\left\|u_{t}\right\|^{2}\right) \leqslant e^{-\varkappa t} \exp \left(\varkappa\|u\|^{2}\right)+C_{2}\left(\varkappa, \mathfrak{B}_{0},\|h\|\right), \\
& \mathbb{E}_{u}\left\{\left\|u_{t}\right\|^{2} \exp \left(\varkappa\left\|u_{t}\right\|^{2}\right)\right\} \leqslant C_{3}\left(t, \varkappa, \mathfrak{B}_{0},\|h\|\right) \exp \left(\varkappa\|u\|^{2}\right),
\end{aligned}
$$

where $\mathcal{E}(t)=\mathcal{E}^{u}(t):=\left\|u_{t}\right\|^{2}+\int_{0}^{t}\left\|u_{s}\right\|_{1}^{2} \mathrm{~d} s$.

Polynomial moments. - For any $m \geqslant 1$,

$$
\begin{aligned}
& \mathbb{E}_{u}\left\|u_{t}\right\|^{2 m} \leqslant e^{-m \alpha_{1} t}\|u\|^{2 m}+C_{4}\left(m, \mathfrak{B}_{0},\|h\|\right), \\
& \mathbb{E}_{u}\left\|u_{t}\right\|_{1}^{2 m} \leqslant C_{5}\left(t, m, \mathfrak{B}_{1},\|h\|_{1}\right)\|u\|^{8 m} .
\end{aligned}
$$

Proof. - Estimate (6.19) is established in Proposition 2.4.10 in [KS12]. To prove $(6.20)$, we denote $\tilde{\mathcal{C}}_{\rho}:=\mathcal{C}_{\rho-1} \backslash \mathcal{C}_{\rho}$, where $\mathcal{C}_{\rho}$ the event on the left-hand side of (6.19) and $\mathcal{C}_{-1}:=\Omega$. Then for any $\varkappa \in\left(0, \gamma_{0}\right)$, we have

$$
\begin{aligned}
\mathbb{E}_{u} e^{\varkappa \mathcal{E}(t)} & =\sum_{\rho=0}^{\infty} \mathbb{E}_{u}\left\{e^{\varkappa \mathcal{E}(t)} I_{\tilde{\mathcal{C}}_{\rho}}\right\} \leqslant e^{\varkappa\left(K t+\|u\|^{2}\right)} \sum_{\rho=0}^{\infty} e^{\varkappa \rho} \mathbb{P}\left\{\mathcal{C}_{\rho-1}\right\} \\
& \leqslant e^{\varkappa\left(K t+\|u\|^{2}\right)+\gamma_{0}} \sum_{\rho=0}^{\infty} e^{\left(\varkappa-\gamma_{0}\right) \rho}=\frac{e^{\varkappa\left(K t+\|u\|^{2}\right)+\gamma_{0}}}{1-e^{\left(\varkappa-\gamma_{0}\right)}} .
\end{aligned}
$$

${ }^{(8)}$ Recall that $\mathfrak{B}_{i}=\sum_{j \geqslant 1} \alpha_{j}^{i} b_{j}^{2}, i=0,1$. 
Estimates (6.21) and (6.24) are proved in Propositions 2.4.9 and 2.4.12 in [KS12], respectively. To show ${ }^{(9)}(6.23)$, we set $F(u)=\|u\|^{2 m}$. Then

$$
\begin{aligned}
& \partial_{u} F(u ; v)=2 m\|u\|^{2(m-1)}\langle u, v\rangle, \\
& \partial_{u}^{2} F(u ; v)=2 m\|u\|^{2(m-1)}\|v\|^{2}+4 m(m-1)\|u\|^{2(m-2)}\langle u, v\rangle^{2},
\end{aligned}
$$

so applying the Itô formula for the functional $F$ and taking the expectation:

$$
\begin{aligned}
\mathbb{E}_{u}\left\|u_{t}\right\|^{2 m}=\|u\|^{2 m}+\mathbb{E}_{u} & \int_{0}^{t} \\
& \left(2 m\left\|u_{s}\right\|^{2(m-1)}\left\langle u_{s},-L u_{s}-B\left(u_{s}\right)+h\right\rangle\right. \\
& \left.+m\left\|u_{s}\right\|^{2(m-1)} \mathfrak{B}_{0}+2 m(m-1)\left\|u_{s}\right\|^{2(m-2)} \sum_{j=1}^{\infty} b_{j}^{2} u_{j}^{2}\right) \mathrm{d} s,
\end{aligned}
$$

where $u_{j}=\left\langle u, e_{j}\right\rangle$. The identity

$$
\langle u, B(u)\rangle=0
$$

and the Cauchy-Schwarz and Poincaré inequalities imply that

$$
\begin{gathered}
\mathbb{E}_{u}\left\|u_{t}\right\|^{2 m} \leqslant\|u\|^{2 m}+\mathbb{E}_{u} \int_{0}^{t}\left(2 m\left\|u_{s}\right\|^{2(m-1)}\left(-\left\|u_{s}\right\|_{1}^{2}+\left\|u_{s}\right\|\|h\|\right)\right. \\
\left.+m\left\|u_{s}\right\|^{2(m-1)} \mathfrak{B}_{0}+2 m(m-1)\left\|u_{s}\right\|^{2(m-1)} \mathfrak{B}_{0}\right) \mathrm{d} s \\
\leqslant\|u\|^{2 m}-m \alpha_{1} \int_{0}^{t} \mathbb{E}_{u}\left\|u_{s}\right\|^{2 m} \mathrm{~d} s+t C_{6}\left(m, \mathfrak{B}_{0},\|h\|\right) .
\end{gathered}
$$

Combining this with the Gronwall inequality, we obtain (6.23).

To prove (6.22), we apply the Itô formula for $F(t, u)=t\|u\|^{2} \exp \left(\varkappa\|u\|^{2}\right)$, use the equalities

$$
\begin{aligned}
& \partial_{t} F(t, u ; v)=\|u\|^{2} \exp \left(\varkappa\|u\|^{2}\right), \\
& \partial_{u} F(t, u ; v)=2 t \exp \left(\varkappa\|u\|^{2}\right)\left(1+\varkappa\|u\|^{2}\right)\langle u, v\rangle, \\
& \partial_{u}^{2} F(t, u ; v)=2 t \exp \left(\varkappa\|u\|^{2}\right)\left(2 \varkappa\left(2+\varkappa\|u\|^{2}\right)\langle u, v\rangle^{2}+\left(1+\varkappa\|u\|^{2}\right)\|v\|^{2}\right),
\end{aligned}
$$

and take the expectation:

$$
\begin{array}{r}
t \mathbb{E}_{u}\left\{\left\|u_{t}\right\|^{2} \exp \left(\varkappa\left\|u_{t}\right\|^{2}\right)\right\}=\mathbb{E}_{u} \int_{0}^{t}\left(\left\|u_{s}\right\|^{2}+2 s\left(1+\varkappa\left\|u_{s}\right\|^{2}\right)\left\langle u_{s},-L u_{s}-B\left(u_{s}\right)+h\right\rangle\right. \\
\left.+s \sum_{j=1}^{\infty}\left[2 \varkappa\left(2+\varkappa\left\|u_{s}\right\|^{2}\right) b_{j}^{2} u_{j}^{2}+\left(1+\varkappa\left\|u_{s}\right\|^{2}\right) b_{j}^{2}\right]\right) \exp \left(\varkappa\left\|u_{s}\right\|^{2}\right) \mathrm{d} s .
\end{array}
$$

${ }^{(9)}$ We confine ourselves to a formal derivation of (6.23). The accurate proof is based on the same arguments applied to the stopped solutions $u\left(t \wedge \tau_{n}\right)$, where $\tau_{n}=\inf \{t \geqslant 0:\|u(t)\|>n\}$. 
Again using (6.25) and the Cauchy-Schwarz and Poincaré inequalities, we get for sufficiently small $\gamma_{0}=\gamma_{0}\left(\mathfrak{B}_{0}\right)>0$ and any $\varkappa \in\left(0, \gamma_{0}\right)$,

$$
\begin{gathered}
t \mathbb{E}_{u}\left\{\left\|u_{t}\right\|^{2} \exp \left(\varkappa\left\|u_{t}\right\|^{2}\right)\right\} \leqslant \mathbb{E}_{u} \int_{0}^{t}\left(\left\|u_{s}\right\|^{2}+2 s\left(1+\varkappa\left\|u_{s}\right\|^{2}\right)\left(-\left\|u_{s}\right\|_{1}^{2}+\left\|u_{s}\right\|\|h\|\right)\right. \\
\left.+s 2 \varkappa\left(2+\varkappa\left\|u_{s}\right\|^{2}\right)\left\|u_{s}\right\|^{2} \mathfrak{B}_{0}+s\left(1+\varkappa\left\|u_{s}\right\|^{2}\right) \mathfrak{B}_{0}\right) \exp \left(\varkappa\left\|u_{s}\right\|^{2}\right) \mathrm{d} s \\
\leqslant \mathbb{E}_{u} \int_{0}^{t}\left(\left\|u_{s}\right\|^{2}+s C_{7}\left(\varkappa, \mathfrak{B}_{0},\|h\|\right)\right) \exp \left(\varkappa\left\|u_{s}\right\|^{2}\right) \mathrm{d} s .
\end{gathered}
$$

Thus (6.22) follows from (6.21), the Poincaré inequality, and the estimate

$$
\mathbb{E}_{u}\left\{\int_{0}^{t}\left\|u_{s}\right\|_{1}^{2} \exp \left(\varkappa\left\|u_{s}\right\|^{2}\right) \mathrm{d} s\right\} \leqslant C_{8}\left(t, \varkappa, \mathfrak{B}_{0},\|h\|\right) \exp \left(\varkappa\|u\|^{2}\right) .
$$

The latter is easily proved by applying the Itô formula for $F(u)=\exp \left(\varkappa\|u\|^{2}\right)$. This completes the proof of the lemma.

\subsection{Hyper-exponential recurrence}

For any $R>0$, let $\tau(R)$ be the first hitting time of the set $X_{R}$ :

$$
\tau(R)=\inf \left\{t \geqslant 0: u_{t} \in X_{R}\right\} .
$$

We have the following standard estimate for the exponential moment of $\tau(R)$.

Proposition 6.4. - For any $\gamma>0$, there are positive numbers $m, R$, and $C$ such that

$$
\mathbb{E}_{u} \exp (\gamma \tau(R)) \leqslant C \mathfrak{w}_{m}(u), \quad u \in H
$$

Proof. - See [JNPS18, Proposition 5.1] for a similar result in the discrete-time case. The proof of (6.27) follows the same arguments. The idea is to establish the inequality for the first hitting time of a ball in $H$ and then to use the regularising property of the Navier-Stokes system.

Step 1: Hyper-exponential recurrence in $H$. - For any $r>0$, we denote by $\tau_{0}(r)$ the first hitting time of the ball $B_{H}(r)$ :

$$
\tau_{0}(r)=\inf \left\{t \geqslant 0: u_{t} \in B_{H}(r)\right\} .
$$

Let us prove that, for any $\gamma>0$, there are positive numbers $m, r$, and $C$ such that

$$
\mathbb{E}_{u} \exp \left(\gamma \tau_{0}(r)\right) \leqslant C \mathfrak{w}_{m}(u), \quad u \in H
$$

Indeed, let $m>0$ be so large that $q:=2 e^{-m \alpha_{1}}<1$. Then, by (6.23), we have

$$
\mathbb{E}_{u}\left\|u_{1}\right\|^{2 m} \leqslant q\left(\|u\|^{2 m} \vee r\right), \quad u \in H,
$$

where $r=e^{m \alpha_{1}} C_{4}\left(m, B_{0},\|h\|\right)$. The Markov property and (6.29) imply that

$$
p_{k}(u):=\mathbb{E}_{u}\left(I_{\left\{\tau_{0}(r)>k\right\}}\left\|u_{k}\right\|^{2 m}\right) \leqslant q^{k}\|u\|^{2 m}, \quad k \geqslant 0, u \in H
$$

(cf. [KS12, proof of Lemma 3.6.1]), hence

$$
\mathbb{P}_{u}\left\{\tau_{0}(r)>k\right\} \leqslant r^{-2 m} p_{k}(u) \leqslant r^{-2 m} q^{k}\|u\|^{2 m} .
$$


As $q<1$, the Borel-Cantelli lemma gives that $\mathbb{P}_{u}\left\{\tau_{0}(r)<\infty\right\}=1$. Choosing $m \geqslant 1$ so large that $e^{\gamma} q<1$, we derive from (6.30)

$$
\begin{aligned}
\mathbb{E}_{u} \exp \left(\gamma \tau_{0}(r)\right) & \leqslant 1+\sum_{k=1}^{\infty} e^{\gamma k} \mathbb{P}_{u}\left\{\tau_{0}(r)>k-1\right\} \\
& \leqslant 1+r^{-2 m}\|u\|^{2 m} \sum_{k=1}^{\infty} e^{\gamma k} q^{k-1} \leqslant C \mathfrak{w}_{m}(u),
\end{aligned}
$$

which proves (6.28).

Step 2: Hyper-exponential recurrence in $H^{1}$. - First note that, for any numbers $p \in(0,1)$ and $r>0$, there is $R>0$ such that

$$
\mathbb{P}_{u}\left\{u_{1} \in X_{R}\right\} \geqslant 1-p, \quad u \in B_{H}(r) .
$$

Indeed, this follows immediately from the Chebyshev inequality and (6.24):

$$
\mathbb{P}_{u}\left\{\left\|u_{1}\right\|_{1}>R\right\} \leqslant R^{-2} \mathbb{E}_{u}\left\|u_{1}\right\|_{1}^{2} \leqslant C R^{-2}\|u\|^{8} \leqslant C R^{-2} r^{8} \leqslant p
$$

for any $u \in B_{H}(r)$ and sufficiently large $R=R(r, p)$.

Now we combine (6.28) and (6.31) to prove (6.27). We introduce the sequences of stopping times

$$
\tau_{0}^{\prime}=\tau_{0}(r), \quad \tau_{n}^{\prime}=\inf \left\{t \geqslant \tau_{n-1}^{\prime}+1: u_{t} \in B_{H}(r)\right\}, \quad n \geqslant 1
$$

and $\tau_{n}=\tau_{n}^{\prime}+1$. Let

$$
\hat{n}=\min \left\{n \geqslant 0: \tau_{n} \in X_{R}\right\} .
$$

From (6.31) and the strong Markov property we have

$$
\mathbb{P}_{u}\{\hat{n}>k\} \leqslant(1-p)^{k}, \quad k \geqslant 0, u \in H,
$$

so $\hat{n}$ is almost surely finite. For any integers $k, M \geqslant 1$, we can write

$$
\begin{aligned}
\mathbb{P}_{u}\{\tau(R) \geqslant M\} & =\mathbb{P}_{u}\left\{\tau(R) \geqslant M, \tau_{k}<M\right\}+\mathbb{P}_{u}\left\{\tau(R) \geqslant M, \tau_{k} \geqslant M\right\} \\
& \leqslant \mathbb{P}_{u}\left\{\tau(R)>\tau_{k}\right\}+\mathbb{P}_{u}\left\{\tau_{k} \geqslant M\right\} .
\end{aligned}
$$

Since $\left\{\tau(R)>\tau_{k}\right\} \subset\{\hat{n}>k\}$, the first probability is estimated by $(1-p)^{k}$. The second one is estimated using (6.28) and the strong Markov property

$$
\mathbb{P}_{u}\left\{\tau_{k} \geqslant M\right\} \leqslant C_{1}^{k} \mathfrak{w}_{m}(u) e^{-3 \gamma M},
$$

where $C_{1}>0$ does not depend on $k, M \geqslant 1$ and $u \in H$. Thus, we obtain

$$
\mathbb{P}_{u}\{\tau(R) \geqslant M\} \leqslant(1-p)^{k}+C_{1}^{k} \mathfrak{w}_{m}(u) e^{-3 \gamma M} .
$$

To complete the proof, it remains to choose appropriately the parameters $k$ and $R$. We take $k \sim \varepsilon M$, where $\varepsilon>0$ is so small that $\varepsilon \log C_{1} \leqslant \gamma$, and $R>0$ so large that $\varepsilon \log (1-p)^{-1} \geqslant 2 \gamma$. Then

$$
\mathbb{P}_{u}\{\tau(R) \geqslant M\} \leqslant 2 e^{-2 \gamma M} \mathfrak{w}_{m}(u),
$$

which implies (6.27). 


\subsection{Generalised Markov semigroups}

For the reader's convenience, we recall here a result on the large-time asymptotics of generalised Markov semigroups in a Polish space $X$. It is established in [JNPS18] in the discrete-time setting, then extended to the continuous-time in [MN18a]. Let us first recall some terminology.

Definition 6.5. - We shall say that $\left\{P_{t}(u, \cdot), u \in X, t \geqslant 0\right\}$ is a generalised Markov family of transition kernels if the following two properties are satisfied.

Feller property. - For any $t \geqslant 0$, the function $u \mapsto P_{t}(u, \cdot)$ is continuous from $X$ to $\mathcal{M}_{+}(X)$ and does not vanish.

Kolmogorov-Chapman relation. - For any $t, s \geqslant 0, u \in X$, and Borel set $\Gamma \subset X$, the following relation holds

$$
P_{t+s}(u, \Gamma)=\int_{X} P_{s}(v, \Gamma) P_{t}(u, \mathrm{~d} v) .
$$

To any such family we associate two semigroups by the following relations:

$$
\begin{array}{rlrl}
\mathfrak{P}_{t}: C_{b}(X) \rightarrow C_{b}(X), & \mathfrak{P}_{t} \psi(u) & =\int_{X} \psi(v) P_{t}(u, \mathrm{~d} v), \\
\mathfrak{P}_{t}^{*}: \mathcal{M}_{+}(X) \rightarrow \mathcal{M}_{+}(X), & \mathfrak{P}_{t}^{*} \mu(\Gamma)=\int_{X} P_{t}(v, \Gamma) \mu(\mathrm{d} v), \quad t \geqslant 0 .
\end{array}
$$

For a measurable function $\mathfrak{w}: X \rightarrow[1,+\infty]$ and a family $\mathcal{C} \subset C_{b}(X)$, we denote by $\mathcal{C}^{\mathfrak{w}}$ the set of functions $\psi \in L_{\mathfrak{w}}^{\infty}(X)$ that can be approximated with respect to the norm $\|\cdot\|_{L_{\mathrm{w}}^{\infty}}$ by finite linear combinations of functions from $\mathcal{C}$. We shall say that a family $\mathcal{C} \subset C_{b}(X)$ is determining if for any $\mu, \nu \in \mathcal{M}_{+}(X)$ satisfying $\langle\psi, \mu\rangle=\langle\psi, \nu\rangle$ for all $\psi \in \mathcal{C}$, we have $\mu=\nu$. Finally, a family of functions $\psi_{t}: X \rightarrow \mathbb{R}$ is uniformly equicontinuous on a subset $K \subset X$ if for any $\varepsilon>0$ there is $\delta>0$ such that $\left|\psi_{t}(u)-\psi_{t}(v)\right|<\varepsilon$ for any $u \in K, v \in B_{X}(u, \delta) \cap K$, and $t \geqslant 0$. The following result is Theorem 7.4 in [MN18a].

Theorem 6.6. - Let $\left\{P_{t}(u, \cdot), u \in X, t \geqslant 0\right\}$ be a generalised Markov family of transition kernels satisfying the following four properties.

Growth conditions. - There is an increasing sequence $\left\{X_{R}\right\}_{R=1}^{\infty}$ of compact subsets of $X$ such that $X_{\infty}:=\bigcup_{R=1}^{\infty} X_{R}$ is dense in $X$. The measures $P_{t}(u, \cdot)$ are concentrated on $X_{\infty}$ for any $u \in X$ and $t>0$, and there is a measurable function $\mathfrak{w}: X \rightarrow[1,+\infty]$ and an integer $R_{0} \geqslant 1$ such that ${ }^{(10)}$

$$
\begin{aligned}
& \sup _{t \geqslant 0} \frac{\left\|\mathfrak{P}_{t} \mathfrak{w}\right\|_{L_{\mathfrak{w}}^{\infty}}}{\left\|\mathfrak{P}_{t} \mathbf{1}\right\|_{R_{0}}}<\infty, \\
& \sup _{t \in[0,1]}\left\|\mathfrak{P}_{t} \mathbf{1}\right\|_{\infty}<\infty,
\end{aligned}
$$

where $\|\cdot\|_{R}$ and $\|\cdot\|_{\infty}$ denote the $L^{\infty}$ norm on $X_{R}$ and $X$, respectively, and we set $\infty / \infty=0$.

Time-continuity. - For any $g \in C_{\mathfrak{w}}(X)$ and $u \in X$, the function $t \mapsto \mathfrak{P}_{t} g(u)$ is continuous from $\mathbb{R}_{+}$to $\mathbb{R}$.

${ }^{(10)}$ The expression $\left(\mathfrak{P}_{t} \mathfrak{w}\right)(u)$ is understood as an integral of a positive function $\mathfrak{w}$ against a positive measure $P_{t}(u, \cdot)$. 
Uniform irreducibility. - For sufficiently large $\rho \geqslant 1$, any $R \geqslant 1$ and $r>0$, there are positive numbers $l=l(\rho, r, R)$ and $p=p(\rho, r)$ such that

$$
P_{l}\left(u, B_{X}(\hat{u}, r)\right) \geqslant p \quad \text { for all } u \in X_{R}, \hat{u} \in X_{\rho} .
$$

Uniform Feller property. - There is a number $R_{0} \geqslant 1$ and a determining family $\mathcal{C} \subset C_{b}(X)$ such that $\mathbf{1} \in \mathcal{C}$ and the family $\left\{\left\|\mathfrak{P}_{t} \mathbf{1}\right\|_{R}^{-1} \mathfrak{P}_{t} \psi, t \geqslant 0\right\}$ is uniformly equicontinuous on $X_{R}$ for any $\psi \in \mathcal{C}$ and $R \geqslant R_{0}$.

Then for any $t>0$, there is at most one measure $\mu_{t} \in \mathcal{P}_{\mathfrak{w}}(X)$ such that $\mu_{t}\left(X_{\infty}\right)=1$ and

$$
\mathfrak{P}_{t}^{*} \mu_{t}=\lambda(t) \mu_{t} \quad \text { for some } \lambda(t) \in \mathbb{R}
$$

satisfying the following condition:

$$
\left\|\mathfrak{P}_{t} \mathfrak{w}\right\|_{R} \int_{X \backslash X_{R}} \mathfrak{w}(u) \mu_{t}(\mathrm{~d} u) \rightarrow 0 \quad \text { as } R \rightarrow \infty .
$$

Moreover, if such a measure $\mu_{t}$ exists for all $t>0$, then it is independent of $t$ (we set $\mu:=\mu_{t}$ ), the corresponding eigenvalue is of the form $\lambda(t)=\lambda^{t}, \lambda>0, \operatorname{supp} \mu=X$, there is a non-negative function $h \in L_{\mathfrak{w}}^{\infty}(X)$ such that $\langle h, \mu\rangle=1$,

$$
\left(\mathfrak{P}_{t} h\right)(u)=\lambda^{t} h(u) \quad \text { for } u \in X, t>0,
$$

the restriction of $h$ to $X_{R}$ belongs to $C_{+}\left(X_{R}\right)$, and for any $\psi \in \mathcal{C}^{\mathfrak{w}}$ and $R \geqslant 1$, we have

$$
\lambda^{-t} \mathfrak{P}_{t} \psi \rightarrow\langle\psi, \mu\rangle h \quad \text { in } C\left(X_{R}\right) \cap L^{1}(X, \mu) \text { as } t \rightarrow \infty .
$$

Finally, if a Borel set $B \subset X$ is such that

$$
\sup _{u \in B} \int_{X \backslash X_{R}} \mathfrak{w}(v) P_{s}(u, \mathrm{~d} v) \rightarrow 0 \quad \text { as } R \rightarrow \infty
$$

for some $s>0$, then

$$
\lambda^{-t} \mathfrak{P}_{t} \psi \rightarrow\langle\psi, \mu\rangle h \quad \text { in } L^{\infty}(B) \text { as } t \rightarrow \infty
$$

for any $\psi \in \mathcal{C}^{\mathfrak{w}}$.

\section{BIBLIOGRAPHY}

[BKL02] Jean Bricmont, Antti Kupiainen, and Raphaël Lefevere, Exponential mixing of the 2D stochastic Navier-Stokes dynamics, Commun. Math. Phys. 230 (2002), no. 1, 87-132. $\uparrow 483$

[DS89] Jean-Dominique Deuschel and Daniel W. Stroock, Large Deviations, Pure and Applied Mathematics, vol. 137, Academic Press Inc., 1989. $\uparrow 483$

[Dud02] Richard M. Dudley, Real analysis and probability, Cambridge Studies in Advanced Mathematics, vol. 74, Cambridge University Press, 2002. $\uparrow 494$

[DV75] Monroe D. Donsker and S. R. Srinivasa Varadhan, Asymptotic evaluation of certain Markov process expectations for large time. I-II, Commun. Pure Appl. Math. 28 (1975), no. 1, 1-47 \& 279-301. 4483

[DZ00] Amir Dembo and Ofer Zeitouni, Large deviations Techniques and applications, Springer, 2000. $\uparrow 483$

[EMS01] Weinan E, Jonathan C. Mattingly, and Yakov G. Sinaŭ, Gibbsian dynamics and ergodicity for the stochastically forced Navier-Stokes equation, Commun. Math. Phys. 224 (2001), no. $1,83-106 . \uparrow 483$ 
[FM95] Franco Flandoli and Bohdan Maslowski, Ergodicity of the 2D Navier-Stokes equation under random perturbations, Commun. Math. Phys. 172 (1995), no. 1, 119-141. 4483

[FP67] Ciprian Foiaš and Giovanni Prodi, Sur le comportement global des solutions nonstationnaires des équations de Navier-Stokes en dimension 2, Rend. Semin. Mat. Univ. Padova 39 (1967), 1-34. $\uparrow 502$

[FW84] Mark I. Freidlin and Alexander D. Wentzell, Random perturbations of dynamical systems, Grundlehren der Mathematischen Wissenschaften, vol. 260, Springer, 1984. $\uparrow 483$

[Gou07a] Mathieu Gourcy, A large deviation principle for 2D stochastic Navier-Stokes equation, Stochastic Processes Appl. 117 (2007), no. 7, 904-927. 4484

[Gou07b] _ Large deviation principle of occupation measure for a stochastic Burgers equation, Ann. Inst. Henri Poincaré, Probab. Stat. 43 (2007), no. 4, 375-408. $\uparrow 484$

[HM06] Martin Hairer and Jonathan C. Mattingly, Ergodicity of the 2D Navier-Stokes equations with degenerate stochastic forcing, Ann. Math. 164 (2006), no. 3, 993-1032. $\uparrow 483,484$

[JNPS15] Vojkan Jakšić, Vahagn Nersesyan, Claude-Alain Pillet, and Armen Shirikyan, Large deviations from a stationary measure for a class of dissipative PDEs with random kicks, Commun. Pure Appl. Math. 68 (2015), no. 12, 2108-2143. ^484, 494

[JNPS18] _ Large deviations and mixing for dissipative PDE's with unbounded random kicks, Nonlinearity 31 (2018), no. 2, 540-596. ^484, 486, 487, 488, 494, 496, 508, 510

[KNS18] Sergei Kuksin, Vahagn Nersesyan, and Armen Shirikyan, Exponential mixing for a class of dissipative PDEs with bounded degenerate noise, 2018. $\uparrow 484$

[KS91] Ioannis Karatzas and Steven E. Shreve, Brownian motion and stochastic calculus, Graduate Texts in Mathematics, vol. 113, Springer, 1991. $\uparrow 482$

[KS00] Sergei Kuksin and Armen Shirikyan, Stochastic dissipative PDEs and Gibbs measures, Commun. Math. Phys. 213 (2000), no. 2, 291-330. $\uparrow 483$

[KS02] _ Coupling approach to white-forced nonlinear PDEs, J. Math. Pures Appl. 81 (2002), no. 6, 567-602. $\uparrow 483$

[KS12] - Mathematics of two-dimensional turbulence, Cambridge Tracts in Mathematics, vol. 194, Cambridge University Press, 2012. ^483, 488, 492, 495, 502, 503, 505, 506, 507, 508

[Kuk02] Sergei B. Kuksin, Ergodic theorems for 2D statistical hydrodynamics, Rev. Math. Phys. 14 (2002), no. 6, 585-600. $\uparrow 483$

[Lio69] Jacques-Louis Lions, Quelques méthodes de résolution des problèmes aux limites non linéaires, Études mathématiques, Dunod, 1969. $\uparrow 482$

[MN18a] Davit Martirosyan and Vahagn Nersesyan, Local large deviations principle for occupation measures of the stochastic damped nonlinear wave equation, Ann. Inst. Henri Poincaré, Probab. Stat. 54 (2018), no. 4, 2002-2041. ^484, 486, 488, 494, 495, 496, 502, 503, 510

[MN18b] _ Multiplicative ergodic theorem for a non-irreducible random dynamical system, 2018. $\uparrow 484,494$

[Oda08] Cyril Odasso, Exponential mixing for stochastic PDEs: the non-additive case, Probab. Theory Relat. Fields 140 (2008), no. 1-2, 41-82. $\uparrow 483$

[Shi06] Armen Shirikyan, Law of large numbers and central limit theorem for randomly forced PDE's, Probab. Theory Relat. Fields 134 (2006), no. 2, 215-247. $\uparrow 483$

[Wu01] Liming $\mathrm{Wu}$, Large and moderate deviations and exponential convergence for stochastic damping Hamiltonian systems, Stochastic Processes Appl. 91 (2001), no. 2, 205-238. $\uparrow 484$ 
Manuscript received on 26th February 2018, revised on 9th February 2019, accepted on 22nd February 2019.

Recommended by Editor A. Debussche. Published under license CC BY 4.0.

(c) (i)

This journal is a member of Centre Mersenne.

MENTRE

Vahagn NERSESYAN

Laboratoire de Mathématiques, UMR CNRS 8100, UVSQ, Université Paris-Saclay

45 Av. des Etats-Unis

78035 Versailles (France)

vahagn.nersesyan@math.uvsq.fr 